\title{
PATTERN SELECTION FOR FARADAY WAVES IN AN INCOMPRESSIBLE VISCOUS FLUID*
}

\author{
A. C. SKELDON ${ }^{\dagger}$ AND G. GUIDOBONI ${ }^{\ddagger}$
}

\begin{abstract}
When a layer of fluid is oscillated up and down with a sufficiently large amplitude, patterns form on the surface, a phenomenon first observed by Faraday. A wide variety of such patterns have been observed from regular squares and hexagons to superlattice and quasipatterns and more exotic patterns such as oscillons. Previous work has investigated the mechanisms of pattern selection using the tools of symmetry and bifurcation theory. The hypotheses produced by these generic arguments have been tested against an equation derived by Zhang and Viñals in the weakly viscous and large depth limit. However, in contrast, many of the experiments use shallow viscous layers of fluid to counteract the presence of high frequency weakly damped modes that can make patterns hard to observe. Here we develop a weakly nonlinear analysis of the full Navier-Stokes equations for the two-frequency excitation Faraday experiment. The problem is formulated for general depth, although results are presented only for the infinite depth limit. We focus on a few particular cases where detailed experimental results exist and compare our analytical results with the experimental observations. Good agreement with the experimental results is found.
\end{abstract}

Key words. Faraday waves, superlattice patterns, weakly nonlinear analysis

AMS subject classification. $37 \mathrm{~N} 10$

DOI. $10.1137 / 050639223$

1. Introduction. Waves on the surface of a fluid excited by a vertical oscillation were first observed by Faraday [1]. Subsequently, in the 1980's, the so-called Faraday crispation experiment became one of the first fluid experiments where mode interactions and chaos were observed [2]. Over the last decade, this experiment has become a testbed for ideas of pattern selection in systems under parametric excitation, and a large variety of patterns have been observed including not just regular patterns of squares and hexagons but many more exotic patterns such as superlattice patterns, quasipatterns, and oscillons. These more recent studies were initiated by the results of Edwards and Fauve [3], who used a two-frequency, rather than a single-frequency, excitation, thereby increasing the number of parameters in the problem and breaking the subharmonic time symmetry. Further two-frequency experiments have been performed by Kudrolli, Pier, and Gollub [4] and Arbell and Fineberg [5, 6, 7]. Subsequently, many of the patterns have been observed in experiments with only a single frequency of excitation [8]. Meanwhile, in practical applications of Faraday waves, the phenomenon has been investigated as a tool to produce patterns on films [9, 10], investigated as a mechanism for transporting gas across an air/water boundary [11], and seen as oscillations on the surface of bubbles [12].

In a container, if the amplitude of the vertical excitation is not too large, then no waves form on the surface of the fluid and the fluid is merely translated up and down. As the amplitude of the excitation is increased, waves appear at a critical amplitude of excitation. This onset of waves was first described theoretically by Benjamin and

\footnotetext{
* Received by the editors August 30, 2005; accepted for publication (in revised form) February 13, 2007; published electronically May 10, 2007.

http://www.siam.org/journals/siap/67-4/63922.html

${ }^{\dagger}$ Department of Mathematics, University of Surrey, Guildford GU2 7XH, UK (a.skeldon@surrey. ac.uk).

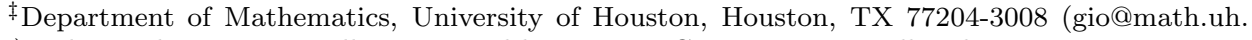
edu). This author was partially supported by a Marie Curie Training Fellowship.
} 
Ursell [13], who showed that for an inviscid infinite layer of fluid the problem reduces to a Mathieu equation. Kumar and Tuckerman [14] developed a method for solving the linear stability problem for the viscous, finite depth fluid problem for a single frequency of excitation. This work was extended to two-frequency excitation [15] and gives excellent agreement with experimental measurements of the onset of patterns.

Understanding not just the onset of patterns but the type of patterns is challenging. The full mathematical description of the fluid problem involves the Navier-Stokes equations in a domain with a free surface, and the excitation makes the problem nonautonomous. Symmetry arguments along with the notion of resonant trial interactions have been used to uncover some of the pattern selection mechanisms [18]. This showed that weakly damped harmonic modes play a key role, with the wavenumber of the weakly damped mode relative to the critical wavenumber being an indicator of what patterns are likely to be seen. Since the wavenumbers of weakly damped modes are determined by the particular forcing function, this in turn has led to theoretical work in the nearly Hamiltonian limit on controlling pattern selection $[19,20]$. In this work, they showed how multiple frequency components in the forcing can be used to enhance particular resonant triad interactions that in turn promote the stability of particular superlattice patterns. The theoretical ideas in $[18,19,20]$ were all tested by calculating the coefficients of the relevant amplitude equations for a two-coupled scalar partial differential equation model derived and analyzed by Zhang and Viñals describing the Faraday problem in a weakly viscous and large depth limit $[16,17]$. While the theory and the results calculated from the Zhang-Vinals equation agree well, it is harder to establish to what degree these pattern selection mechanisms can be used to explain experimental findings. This is because many of the experimental studies use a fluid that is either moderately viscous or a container that is shallow, neither of which is within the range of validity of the Zhang-Viñals model. The reason that experiments tend to focus on these cases is because of the presence of long wavelength modes that can make it difficult to observe regular patterns: these modes can be damped either by increasing the viscosity or by increasing the dissipation from the lower boundary by making the container shallower [21]. The large viscosity also minimizes the impact of the lateral boundaries on the patterns and the effect of patterns formed by meniscus waves emitted from the sidewalls.

Weakly nonlinear analysis from the full fluid equations for single-frequency excitation in an infinite fluid layer has been carried out by Chen and Viñals [22]. In this paper, we extend the formulation of the weakly nonlinear problem to two-frequency excitation and to finite fluid depth. The former involves a significantly different approach to the derivation of a solvability condition: for a single frequency of excitation, the form of the linear problem may be written as a recursion relation, and an adjoint to this recursion relation may be defined. This works because, in the linear Faraday problem, modes with different frequencies are coupled only through the excitation. Specifically, a frequency component $\cos \omega t$ in the excitation couples the $n$th Fourier mode to the $n-1$ and the $n+1$ mode. When an $N$ mode truncation is taken, then the equation for the $N$ th mode is coupled only to the $(N-1)$ th mode. Consequently, one can solve for the $N$ th mode in terms of the $(N-1)$ th mode. In turn, this then allows one to solve for the $(N-1)$ th mode and, recursively, for all modes. If instead multiple frequency forcing, for example, $\cos \chi \cos M_{1} \omega t+\sin \chi \cos M_{2} \omega t$, is used, then the $n$th Fourier mode is coupled to four other modes, $n-M_{1}, n-M_{2}, n+M_{1}, n+M_{2}$. Truncating at $N$ modes leaves the $N$ th mode coupled to both $N-M_{1}$ and $N-M_{2}$, and so a recursion relation cannot readily be defined.

In the future, we will investigate the effect of depth on the coefficients of the 
amplitude equations; however, for the purposes of this paper, we have focused on carrying out the calculations and detailed results for infinite depth only.

The pattern selection problem is further complicated by several issues. First, above onset not just a single wavenumber but a band of wavenumbers is unstable. Allowing for variation of the spatial scale to account for this typically leads to Ginzburg-Landau-type amplitude equations. Second, in the viscous Faraday problem, there are weakly damped long wavelength modes. These are coupled to the free surface deformation so that the larger the amplitude of the Faraday waves the more significant the effect. Both of these effects are discussed in [23] for Faraday waves in two space dimensions, but as yet there has been no attempt to include these effects in three space dimensions. Note that since the weakly damped long wavelength modes are coupled to the surface deformation, they do not effect the pattern selection at onset but could have an effect thereafter. Finally, at onset the wavenumber specifies the magnitude but not the direction of the associated wavevector. The spatial scale is therefore determined but not the particular pattern. Typically, a finite number of wavevectors are considered and amplitude equations derived for the amplitude associated with each wavevector. Two approaches are taken. In the first, an integer number of eigenvectors corresponding to modes that are equispaced around a circle are considered. Depending on the number of modes used, this leads to an amplitude equation describing squares, hexagons, or quasipatterns. The amplitude equations are of gradient form, and a Lyapunov function can be written down. The relative stability of the different patterns is then inferred from the relative value of the energy for the different states. A clear discussion of some of the issues involved in using amplitude equations to describe quasipatterns is given in [24]. Alternatively, eigenvectors that generate different spatially periodic lattices are considered. Amplitude equations may again be derived, but this time the eigenvalues indicating the relative stability for different patterns that are supported by the same lattice are considered. The methods are closely related, as discussed further in sections 4 and 6 . In their single-frequency study, Chen and Vinãls [22] focus on squares, hexagons, and quasipatterns. Here in our two-frequency approach we, at least initially, consider spatially periodic patterns on a lattice. We apply our results to the particular two-frequency experimental results of Kudrolli, Pier, and Gollub [4] and find good agreement with their observations.

The layout of this paper is as follows. In section 2, we set up the mathematical problem. In section 3, a weakly nonlinear expansion about the critical wavenumber is carried out and the weakly nonlinear equations at each order derived. In section 4, we briefly discuss the pattern formation context within which we work and specify the general form of the solutions in the horizontal direction. This leads to a sequence of problems for the surface height and the vertical dependence of the velocity. These equations are solved in section 5, leading to the evaluation of the coefficients for the amplitude equations describing the weakly nonlinear pattern formation. The problem contains a number of physical parameters, and the coefficients are calculated for a range of values relevant to the experimental results in [4]. The calculations in section 5 are performed only in the case of infinite depth, although all early sections are not restricted in this way. The justification for this and the implications of the values of the coefficients for the pattern selection are discussed in section 6. Our conclusions are drawn in section 7 .

2. Mathematical model. We consider an infinite horizontal layer of viscous incompressible fluid of finite depth that is subjected to gravity $g$ and to a vertical periodic acceleration of amplitude $a$. At the lower boundary the fluid is in contact 


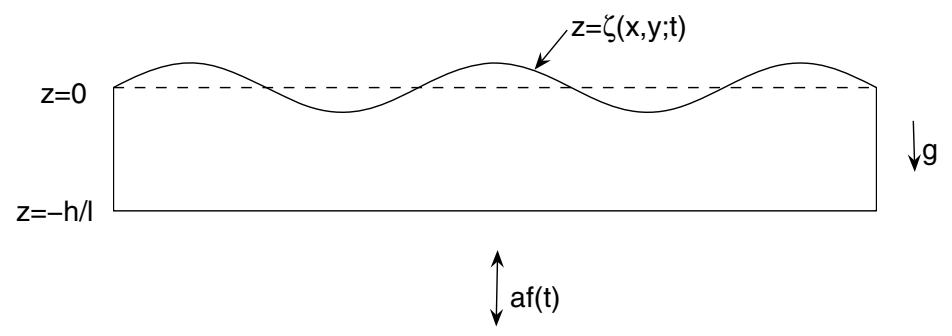

FIG. 2.1. Sketch of a cross-section through the layer of fluid.

with a rigid plane, while at the upper boundary the surface is open to the external ambient conditions. This means that the upper surface is a free boundary whose shape and evolution is an unknown of the problem.

We consider a frame of reference which is moving with the periodic excitation whose $z$-axis is perpendicular to the rigid plane at the bottom at $z=-h / l$, where $h / l$ is the nondimensional depth of the layer. A sketch of the geometry is shown in Figure 2.1. We suppose the free surface is regular enough to be written in the Cartesian representation $z=\zeta(x, y ; t)$; then the fluid motion is described by the dimensionless Navier-Stokes equations

$$
\begin{aligned}
\nabla \cdot \mathbf{u} & =0, \\
\partial_{t} \mathbf{u}+\mathbf{u} \cdot \nabla \mathbf{u} & =-\nabla \mathcal{P}+C \Delta \mathbf{u}-(1+a f(t)) \mathbf{e}_{3},
\end{aligned}
$$

where $\mathbf{u}=(u, v, w)$ is the velocity field, $\mathcal{P}$ the pressure, and

$$
f(t) \equiv f_{1}(t)=\cos (\omega t)
$$

for single-frequency excitation and

$$
f(t)=f_{2}(t)=\cos (\chi) \cos \left(M_{1} \omega t\right)+\sin (\chi) \cos \left(M_{2} \omega t+\phi\right)
$$

for two-frequency excitation, where $M_{1}$ and $M_{2}$ are integers and $\chi$ and $\phi$ are real. The units of length, time, velocity, and pressure have been taken as $l, \sqrt{l / g}, \sqrt{g l}$, and $\varrho g l$, respectively. The amplitude of the acceleration due to the excitation, $a$, is measured in units of $g$. Here $l$ is taken as $k_{c}^{-1}$, where $k_{c}$ is the wavenumber of the pattern at onset. The parameter $C=\nu /\left(g l^{3}\right)^{1 / 2}$ is the square of the inverse of the Galileo number, where $\nu$ is the kinematic viscosity of the fluid. We have used the notation $\nabla=\left(\nabla_{H}, \partial_{z}\right)$, with $\nabla_{H}=\left(\partial_{x}, \partial_{y}\right)$. Equations (2.1) apply in a domain $\Omega=\Sigma \times(-h / l, \zeta(x, y ; t))$, where $\Sigma$ is the horizontal periodicity cell. The bottom of the container, at $z=-h / l$, is rigid, and therefore we take no-slip boundary conditions here:

$$
u=v=w=0 .
$$

At the free surface $z=\zeta(x, y ; t)$ we have the kinematic condition, which says that the surface is advected by the fluid, and two further conditions, one for the balance of the tangential stresses and one for the balance of normal stresses. This leads to three conditions at $z=\zeta(x, y ; t)$, namely

$$
\begin{aligned}
\partial_{t} \zeta+u \partial_{x} \zeta+v \partial_{y} \zeta & =w \\
\mathbf{t}_{1} \cdot \mathbf{T n}=\mathbf{t}_{2} \cdot \mathbf{T n} & =0 \\
-\mathcal{P}+2 C \mathbf{n D}(\mathbf{u}) \mathbf{n} & =B \mathcal{H}-p_{e},
\end{aligned}
$$


where $\mathbf{T}=-\mathcal{P} \mathbf{I}+2 C \mathbf{D}(\mathbf{u})$ is the stress tensor, $\mathbf{D}(\mathbf{u})=\left(\nabla \mathbf{u}+\nabla^{T} \mathbf{u}\right) / 2$ is the rate-ofstrain tensor, $\mathcal{H}=\nabla_{H} \cdot\left(\nabla_{H} \zeta / \sqrt{1+\left|\nabla_{H} \zeta\right|^{2}}\right)$ is the double mean curvature, and the unit normal and tangent vectors are defined as

$$
\begin{aligned}
& \mathbf{n}(x, y ; t)=\left(-\frac{\partial_{x} \zeta}{\sqrt{1+\left|\nabla_{H} \zeta\right|^{2}}},-\frac{\partial_{y} \zeta}{\sqrt{1+\left|\nabla_{H} \zeta\right|^{2}}}, \frac{1}{\sqrt{1+\left|\nabla_{H} \zeta\right|^{2}}}\right) \\
& \mathbf{t}_{1}(x, y ; t)=\left(\frac{1}{\sqrt{1+\left|\partial_{x} \zeta\right|^{2}}}, 0, \frac{\partial_{x} \zeta}{\sqrt{1+\left|\partial_{x} \zeta\right|^{2}}}\right) \\
& \mathbf{t}_{2}(x, y ; t)=\left(0, \frac{1}{\sqrt{1+\left|\partial_{y} \zeta\right|^{2}}}, \frac{\partial_{y} \zeta}{\sqrt{1+\left|\partial_{y} \zeta\right|^{2}}}\right) .
\end{aligned}
$$

Here $p_{e}$ is the dimensionless pressure of the external ambient fluid and is assumed known. The parameter $B=\sigma / \varrho g l^{2}$, where $\sigma$ is the surface tension and $\varrho$ the density of the fluid, is the inverse Bond number and is a nondimensional measure of the relative importance of surface tension and gravity.

It is convenient to define a new pressure,

$$
p=\mathcal{P}+(1+a f(t)) z,
$$

and this has the effect of shifting the acceleration term from the momentum equation to the normal stress condition. In addition, we eliminate the pressure from the momentum equation by taking $-(\nabla \times \nabla \times)$. Using the relation $\nabla \times \nabla \times \mathbf{u}=\nabla(\nabla \cdot \mathbf{u})-\Delta \mathbf{u}$ and the fact that $\nabla \cdot \mathbf{u}=0$, the problem then becomes

$$
\begin{aligned}
\nabla \cdot \mathbf{u} & =0 \\
\partial_{t} \Delta \mathbf{u}-C \Delta \Delta \mathbf{u} & =\nabla \times \nabla \times(\mathbf{u} \cdot \nabla \mathbf{u}),
\end{aligned}
$$

with boundary conditions on $z=-h / l$,

$$
u=v=w=0
$$

and on $z=\zeta$,

$$
\begin{aligned}
\partial_{t} \zeta+u \partial_{x} \zeta+v \partial_{y} \zeta & =w, \\
\mathbf{t}_{1} \cdot \mathbf{T n}=\mathbf{t}_{2} \cdot \mathbf{T n} & =0, \\
2 C \mathbf{n D}(\mathbf{u}) \mathbf{n} & =B \mathcal{H}+p-p_{e}-(1+a f(t)) \zeta .
\end{aligned}
$$

Equations (2.7) with boundary conditions (2.8) and (2.9) have a trivial solution,

$$
\mathbf{u}=\mathbf{0}, \quad p=p_{e}, \quad \zeta=0 .
$$

This solution corresponds to a flat-surface state where there is no relative motion of the fluid with respect to the moving frame.

3. Weakly nonlinear analysis. The flat-surface state loses stability at a critical amplitude of the excitation frequency to regular patterns of standing waves. We use a multiple timescale approach to derive equations describing the amplitude of these standing waves near threshold. In order to do this, the governing equations and the boundary conditions are expanded in a power series of the dimensionless distance 
away from the threshold, $\varepsilon$, and solved order by order in $\varepsilon$. So for the driving dimensionless amplitude $a$, we let $a=a_{0}+\varepsilon a_{1}+\varepsilon^{2} a_{2}$ and expand the flow variables as

$$
\begin{aligned}
\mathbf{u} & =\varepsilon \mathbf{u}_{1}+\varepsilon^{2} \mathbf{u}_{2}+\varepsilon^{3} \mathbf{u}_{3}+\cdots, \\
p & =p_{e}+\varepsilon p_{1}+\varepsilon^{2} p_{2}+\varepsilon^{3} p_{3}+\cdots, \\
\zeta & =\varepsilon \zeta_{1}+\varepsilon^{2} \zeta_{2}+\varepsilon^{3} \zeta_{3}+\cdots
\end{aligned}
$$

At each order in $\varepsilon$ the solution is defined in a different domain since each $\zeta_{i}$ is different. In order to overcome this difficulty, Chen and Vinãls [22] take a Taylor expansion of the boundary conditions at the free surface around the flat surface state $z=0$, so that they consider the solution in $\Sigma \times[-h / l, 0]$ at each order. We follow the same approach here. Near threshold, $\varepsilon \ll 1$, we separate fast and slow timescales: $t=\tau+T_{1} / \varepsilon+T_{2} / \varepsilon^{2}$ such that $\partial_{t}=\partial_{\tau}+\varepsilon \partial_{T_{1}}+\varepsilon^{2} \partial_{T_{2}}$. The fast timescale is the timescale of the excitation, while the slower timescales describe the evolution of the amplitude of the patterns over many periods of the excitation. In sections $3.1,3.2$, and 3.3 , we list the problem for each of the first three orders in $\varepsilon$. These agree with those used in the computations of [22], although note that there is a typographical error in their paper for the normal stress boundary condition at third order. In section 3.4, we derive the linear adjoint problem that is needed in order to find the solvability conditions that lead to the amplitude equations. The general form for the solvability conditions themselves are given in section 3.5. As found in [14] for the linear problem, the linear operator on the left-hand side of the hierarchy of problems for different $\varepsilon$ depends only on the vertical velocity $w$ and on the height of surface $\zeta$. The horizontal components of the velocity, $u$ and $v$, and the pressure, $p$, are needed to evaluate the nonlinear terms that appear on the right-hand side. These may be computed from $w$ and $\zeta$ : details are given in the appendices.

\subsection{Linear problem (first order problem).}

$$
\partial_{\tau} \Delta w_{1}-C \Delta \Delta w_{1}=0,
$$

with boundary conditions on $z=-h / l$,

$$
w_{1}=\partial_{z} w_{1}=0
$$

and on $z=0$,

$$
\begin{aligned}
\partial_{\tau} \zeta_{1}-w_{1} & =0 \\
\Delta_{H} w_{1}-\partial_{z}^{2} w_{1} & =0 \\
-\partial_{\tau} \partial_{z} w_{1}+C \partial_{z}^{3} w_{1}+3 C \Delta_{H} \partial_{z} w_{1} & \\
-B \Delta_{H} \Delta_{H} \zeta_{1}+\left(1+a_{0} f(\tau)\right) \Delta_{H} \zeta_{1} & =0 .
\end{aligned}
$$

Here $\Delta_{H}=\partial_{x}^{2}+\partial_{y}^{2}$.

\subsection{Second order problem.}

$$
\partial_{\tau} \Delta w_{2}-C \Delta \Delta w_{2}=N_{e q}^{(2)},
$$

with boundary conditions on $z=-h / l$,

$$
w_{2}=\partial_{z} w_{2}=0
$$


and on $z=0$,

$$
\begin{aligned}
\partial_{\tau} \zeta_{2}-w_{2} & =N_{k c}^{(2)}, \\
\Delta_{H} w_{2}-\partial_{z}^{2} w_{2} & =N_{t s}^{(2)}, \\
-\partial_{\tau} \partial_{z} w_{2}+C \partial_{z}^{3} w_{2}+3 C \Delta_{H} \partial_{z} w_{2} & \\
-B \Delta_{H} \Delta_{H} \zeta_{2}+\left(1+a_{0} f(\tau)\right) \Delta_{H} \zeta_{2} & =N_{n s}^{(2)} .
\end{aligned}
$$

Here

$$
\begin{aligned}
N_{e q}^{(2)}= & {\left[\nabla \times \nabla \times\left(\mathbf{u}_{1} \cdot \nabla\right) \mathbf{u}_{1}\right] \cdot \mathbf{e}_{3}-\partial_{T_{1}} \Delta w_{1} } \\
N_{k c}^{(2)}= & -\partial_{T_{1}} \zeta_{1}-u_{1} \partial_{x} \zeta_{1}-v_{1} \partial_{y} \zeta_{1}+\partial_{z} w_{1} \zeta_{1} \\
N_{t s}^{(2)}= & \partial_{x}\left[-\partial_{z z} u_{1} \zeta_{1}-\partial_{x z} w_{1} \zeta_{1}+2\left(\partial_{x} u_{1}-\partial_{z} w_{1}\right) \partial_{x} \zeta_{1}+\left(\partial_{y} u_{1}+\partial_{x} v_{1}\right) \partial_{y} \zeta_{1}\right] \\
& +\partial_{y}\left[-\partial_{z z} v_{1} \zeta_{1}-\partial_{y z} w_{1} \zeta_{1}+2\left(\partial_{y} v_{1}-\partial_{z} w_{1}\right) \partial_{y} \zeta_{1}+\left(\partial_{y} u_{1}+\partial_{x} v_{1}\right) \partial_{x} \zeta_{1}\right], \\
N_{n s}^{(2)}= & \partial_{T_{1}} \partial_{z} w_{1}-\nabla_{H} \cdot\left(\mathbf{u}_{1} \cdot \nabla\right) \mathbf{u}_{1}+\Delta_{H}\left(-2 C \partial_{z z} w_{1} \zeta_{1}+\partial_{z} p_{1} \zeta_{1}\right)-a_{1} f(\tau) \Delta_{H} \zeta_{1} .
\end{aligned}
$$

\subsection{Third order problem.}

$$
\partial_{\tau} \Delta w_{3}-C \Delta \Delta w_{3}=N_{e q}^{(3)},
$$

with boundary conditions on $z=-h / l$,

$$
w_{3}=\partial_{z} w_{3}=0
$$

and on $z=0$,

$$
\begin{aligned}
\partial_{\tau} \zeta_{3}-w_{3} & =N_{k c}^{(3)} \\
\Delta_{H} w_{3}-\partial_{z}^{2} w_{3} & =N_{t s}^{(3)} \\
-\partial_{\tau} \partial_{z} w_{3}+C \partial_{z}^{3} w_{3}+3 C \Delta_{H} \partial_{z} w_{3} & \\
-B \Delta_{H} \Delta_{H} \zeta_{3}+\left(1+a_{0} f(\tau)\right) \Delta_{H} \zeta_{3} & =N_{n s}^{(3)}
\end{aligned}
$$

Here

$$
\begin{aligned}
N_{e q}^{(3)}= & {\left[\nabla \times \nabla \times\left(\mathbf{u}_{1} \cdot \nabla\right) \mathbf{u}_{2}\right] \cdot \mathbf{e}_{3}+\left[\nabla \times \nabla \times\left(\mathbf{u}_{2} \cdot \nabla\right) \mathbf{u}_{1}\right] \cdot \mathbf{e}_{3}-\partial_{T_{2}} \Delta w_{1}-\partial_{T_{1}} \Delta w_{2}, } \\
N_{k c}^{(3)}= & -\partial_{T_{2}} \zeta_{1}-\partial_{T_{1}} \zeta_{2}+\partial_{z} w_{1} \zeta_{2}+\partial_{z} w_{2} \zeta_{1}+\frac{1}{2} \partial_{z z} w_{1} \zeta_{1}^{2} \\
& -u_{1} \partial_{x} \zeta_{2}-u_{2} \partial_{x} \zeta_{1}-\partial_{z} u_{1} \zeta_{1} \partial_{x} \zeta_{1}-v_{1} \partial_{y} \zeta_{2}-v_{2} \partial_{y} \zeta_{1}-\partial_{z} v_{1} \zeta_{1} \partial_{y} \zeta_{1} \\
N_{t s}^{(3)}= & \partial_{x}\left[-\partial_{z z} u_{2} \zeta_{1}-\partial_{z z} u_{1} \zeta_{2}-\frac{1}{2} \partial_{z z z} u_{1} \zeta_{1}^{2}-\partial_{x z} w_{2} \zeta_{1}-\partial_{x z} w_{1} \zeta_{2}-\frac{1}{2} \partial_{x z z} w_{1} \zeta_{1}^{2}\right. \\
& -2\left(\partial_{z} w_{2}-\partial_{x} u_{2}\right) \partial_{x} \zeta_{1}-2\left(\partial_{z} w_{1}-\partial_{x} u_{1}\right) \partial_{x} \zeta_{2}-2 \partial_{z}\left(\partial_{z} w_{1}-\partial_{x} u_{1}\right) \zeta_{1} \partial_{x} \zeta_{1} \\
& \left.+\left(\partial_{y} u_{2}+\partial_{x} v_{2}\right) \partial_{y} \zeta_{1}+\left(\partial_{y} u_{1}+\partial_{x} v_{1}\right) \partial_{y} \zeta_{2}+\partial_{z}\left(\partial_{y} u_{1}+\partial_{x} v_{1}\right) \zeta_{1} \partial_{y} \zeta_{1}\right] \\
& +\partial_{y}\left[-\partial_{z z} v_{2} \zeta_{1}-\partial_{z z} v_{1} \zeta_{2}-\frac{1}{2} \partial_{z z z} v_{1} \zeta_{1}^{2}-\partial_{y z} w_{2} \zeta_{1}-\partial_{y z} w_{1} \zeta_{2}-\frac{1}{2} \partial_{y z z} w_{1} \zeta_{1}^{2}\right. \\
& -2\left(\partial_{z} w_{2}-\partial_{y} v_{2}\right) \partial_{y} \zeta_{1}-2\left(\partial_{z} w_{1}-\partial_{y} v_{1}\right) \partial_{y} \zeta_{2}-2 \partial_{z}\left(\partial_{z} w_{1}-\partial_{y} v_{1}\right) \zeta_{1} \partial_{y} \zeta_{1} \\
& \left.+\left(\partial_{y} u_{2}+\partial_{x} v_{2}\right) \partial_{x} \zeta_{1}+\left(\partial_{y} u_{1}+\partial_{x} v_{1}\right) \partial_{x} \zeta_{2}+\partial_{z}\left(\partial_{y} u_{1}+\partial_{x} v_{1}\right) \zeta_{1} \partial_{x} \zeta_{1}\right]
\end{aligned}
$$




$$
\begin{aligned}
N_{n s}^{(3)}= & \partial_{T_{2}} \partial_{z} w_{1}+\partial_{T_{1}} \partial_{z} w_{2}-a_{2} f(\tau) \Delta_{H} \zeta_{1}-a_{1} f(\tau) \Delta_{H} \zeta_{2}-\nabla_{H} \cdot\left[\mathbf{u}_{1} \cdot \nabla \mathbf{u}_{2}+\mathbf{u}_{2} \cdot \nabla \mathbf{u}_{1}\right] \\
& +\Delta_{H}\left[\partial_{z} p_{2} \zeta_{1}+\partial_{z} p_{1} \zeta_{2}+\frac{1}{2} \partial_{z z} p_{1} \zeta_{1}^{2}-2 C \partial_{z z} w_{1} \zeta_{2}-2 C \partial_{z z} w_{2} \zeta_{1}-C \partial_{z z z} w_{1} \zeta_{1}^{2}\right. \\
& +2 C\left(\partial_{z} u_{2}+\partial_{x} w_{2}\right) \partial_{x} \zeta_{1}+2 C\left(\partial_{z} w_{1}-\partial_{x} u_{1}\right)\left(\partial_{x} \zeta_{1}\right)^{2}+2 C\left(\partial_{z} v_{2}+\partial_{y} w_{2}\right) \partial_{y} \zeta_{1} \\
& +2 C \partial_{z}\left(\partial_{x} w_{1}+\partial_{z} u_{1}\right) \partial_{x} \zeta_{1} \zeta_{1}+2 C \partial_{z}\left(\partial_{y} w_{1}+\partial_{z} v_{1}\right) \partial_{y} \zeta_{1} \zeta_{1} \\
& +2 C\left(\partial_{z} w_{1}-\partial_{y} v_{1}\right)\left(\partial_{y} \zeta_{1}\right)^{2}-2 C\left(\partial_{y} u_{1}+\partial_{x} v_{1}\right) \partial_{x} \zeta_{1} \partial_{y} \zeta_{1}-\frac{3}{2} B \partial_{x x} \zeta_{1}\left(\partial_{x} \zeta_{1}\right)^{2} \\
& \left.-\frac{3}{2} B \partial_{y y} \zeta_{1}\left(\partial_{y} \zeta_{1}\right)^{2}-\frac{1}{2} B \partial_{x x} \zeta_{1}\left(\partial_{y} \zeta_{1}\right)^{2}-\frac{1}{2} B \partial_{y y} \zeta_{1}\left(\partial_{x} \zeta_{1}\right)^{2}-2 B \partial_{x} \zeta_{1} \partial_{y} \zeta_{1} \partial_{x y} \zeta_{1}\right]
\end{aligned}
$$

3.4. Linear adjoint problem. In order to use the Fredholm alternative and derive a solvability condition, the solution to the linear adjoint problem is needed. We suppose that $S_{1}=\left(w_{1}, \zeta_{1}\right)$ is the solution of the linear problem and denote by $S^{*}=\left(w^{*}, \zeta^{*}\right)$ the solution of the linear adjoint problem. Then $S_{1}$ and $S^{*}$ satisfy

$$
\left(S^{*}, \mathcal{L} S_{1}\right)=0=\left(\mathcal{L}^{*} S^{*}, S_{1}\right)
$$

where $\mathcal{L}$ and $\mathcal{L}^{*}$ are the linear and the linear adjoint operators, respectively, and $(\cdot, \cdot)$ means the following scalar product:

$$
\int_{0}^{2 \pi / \omega} \int_{\Omega} w^{*}\left(\partial_{\tau} \Delta w_{1}-C \Delta \Delta w_{1}\right) d \Omega d \tau+\int_{0}^{2 \pi / \omega} \int_{\Sigma} \zeta_{*}\left[\partial_{\tau} \zeta_{1}-w_{1}\right]_{z=0} d \Sigma d \tau=0
$$

where $\Omega=\Sigma \times(-h / l, 0)$ and $\Sigma$ is the horizontal periodicity cell.

3.5. Solvability conditions. From the Fredholm alternative theorem it follows that at second order the solvability condition takes the form of

$$
\begin{aligned}
\int_{0}^{2 \pi / \omega} & \int_{\Omega} w^{*}\left(\partial_{\tau} \Delta w_{2}-C \Delta \Delta w_{2}-N_{e q}^{(2)}\right) d \Omega d \tau \\
& +\int_{0}^{2 \pi / \omega} \int_{\Sigma}\left[\zeta^{*}\left(\partial_{\tau} \zeta_{2}-w_{2}-N_{k c}^{(2)}\right)\right]_{z=0} d \Sigma d \tau=0 .
\end{aligned}
$$

This implies that

$$
\begin{gathered}
\int_{0}^{2 \pi / \omega} \int_{\Omega} w^{*} N_{e q}^{(2)} d \Omega d \tau+\int_{0}^{2 \pi / \omega} \int_{\Sigma}\left[\zeta^{*} N_{k c}^{(2)}\right]_{z=0} d \Sigma d \tau \\
+\int_{0}^{2 \pi / \omega} \int_{\Sigma}\left[w^{*} N_{n s}^{(2)}\right]_{z=0} d \Sigma d \tau+C \int_{0}^{2 \pi / \omega} \int_{\Sigma}\left[\partial_{z} w^{*} N_{t s}^{(2)}\right]_{z=0} d \Sigma d \tau=0 .
\end{gathered}
$$

Similarly at third order, we have 


$$
\begin{gathered}
\int_{0}^{2 \pi / \omega} \int_{\Omega} w^{*} N_{e q}^{(3)} d \Omega d \tau+\int_{0}^{2 \pi / \omega} \int_{\Sigma}\left[\zeta^{*} N_{k c}^{(3)}\right]_{z=0} d \Sigma d \tau \\
+\int_{0}^{2 \pi / \omega} \int_{\Sigma}\left[w^{*} N_{n s}^{(3)}\right]_{z=0} d \Sigma d \tau+C \int_{0}^{2 \pi / \omega} \int_{\Sigma}\left[\partial_{z} w^{*} N_{t s}^{(3)}\right]_{z=0} d \Sigma d \tau=0 .
\end{gathered}
$$

4. Patterns. In order to proceed further, we need to first solve the linear problem set out in section 3.1. However, in an unbounded horizontal domain, while the linear problem predicts the onset of spatially periodic patterns at a given excitation frequency and excitation amplitude with a wavenumber $k_{c}$, it does not uniquely determine the pattern that is produced. This is related to the fact that, in an unbounded horizontal domain, the Faraday problem is isotropic so that no particular direction is preferred: any wavevector with wavenumber $k_{c}$ would give an allowable solution; for example, stripes with any orientation would be possible. Furthermore, within the linear problem, linear superposition of different wavevectors with the critical wavenumber also give solutions. In this way, solutions such as squares, hexagons, superlattice patterns, and quasipatterns may be constructed by adding together stripe solutions of the appropriate orientation. However, the fact that these are solutions to the linear problem does not guarantee their existence or stability for the nonlinear problem. Indeed, only particular combinations of patterns are observed in experiments. Here we consider patterns that are spatially periodic, and this is implicit in our choice of domain in sections 2 and 3.

For patterns that are spatially periodic in two space dimensions, previous work has used equivariant bifurcation theory to find the generic types of solutions that exist [25], the generic amplitude equations that these patterns satisfy, and the stability of each pattern in terms of the coefficients of these amplitude equations [26]. For example, on the family of lattices with hexagonal symmetry, the generic amplitude equations are

$$
\begin{aligned}
\dot{z}_{1}= & \lambda z_{1}+\epsilon \bar{z}_{2} \bar{z}_{3} \\
& +\left(b_{1}\left|z_{1}\right|^{2}+b_{2}\left|z_{2}\right|^{2}+b_{2}\left|z_{3}\right|^{2}+b_{4}\left|z_{4}\right|^{2}+b_{5}\left|z_{5}\right|^{2}+b_{6}\left|z_{6}\right|^{2}\right) z_{1}+\mathcal{O}\left(|\mathbf{z}|^{4}\right), \\
\dot{z}_{2}= & \lambda z_{2}+\epsilon \bar{z}_{3} \bar{z}_{1} \\
& +\left(b_{2}\left|z_{1}\right|^{2}+b_{1}\left|z_{2}\right|^{2}+b_{2}\left|z_{3}\right|^{2}+b_{6}\left|z_{4}\right|^{2}+b_{4}\left|z_{5}\right|^{2}+b_{5}\left|z_{6}\right|^{2}\right) z_{2}+\mathcal{O}\left(|\mathbf{z}|^{4}\right), \\
\dot{z}_{3}= & \lambda z_{3}+\epsilon \bar{z}_{1} \bar{z}_{2} \\
& +\left(b_{2}\left|z_{1}\right|^{2}+b_{2}\left|z_{2}\right|^{2}+b_{1}\left|z_{3}\right|^{2}+b_{5}\left|z_{4}\right|^{2}+b_{6}\left|z_{5}\right|^{2}+b_{4}\left|z_{6}\right|^{2}\right) z_{3}+\mathcal{O}\left(|\mathbf{z}|^{4}\right), \\
\dot{z}_{4}= & \lambda z_{4}+\epsilon \bar{z}_{6} \bar{z}_{5} \\
& +\left(b_{4}\left|z_{1}\right|^{2}+b_{6}\left|z_{2}\right|^{2}+b_{5}\left|z_{3}\right|^{2}+b_{1}\left|z_{4}\right|^{2}+b_{2}\left|z_{5}\right|^{2}+b_{2}\left|z_{6}\right|^{2}\right) z_{4}+\mathcal{O}\left(|\mathbf{z}|^{4}\right), \\
\dot{z}_{5}= & \lambda z_{5}+\epsilon \bar{z}_{4} \bar{z}_{6} \\
& +\left(b_{5}\left|z_{1}\right|^{2}+b_{4}\left|z_{2}\right|^{2}+b_{6}\left|z_{3}\right|^{2}+b_{2}\left|z_{4}\right|^{2}+b_{1}\left|z_{5}\right|^{2}+b_{2}\left|z_{6}\right|^{2}\right) z_{5}+\mathcal{O}\left(|\mathbf{z}|^{4}\right), \\
\dot{z}_{6}= & \lambda z_{6}+\epsilon \bar{z}_{5} \bar{z}_{4} \\
& +\left(b_{6}\left|z_{1}\right|^{2}+b_{5}\left|z_{2}\right|^{2}+b_{4}\left|z_{3}\right|^{2}+b_{2}\left|z_{4}\right|^{2}+b_{2}\left|z_{5}\right|^{2}+b_{1}\left|z_{6}\right|^{2}\right) z_{6}+\mathcal{O}\left(|\mathbf{z}|^{4}\right),
\end{aligned}
$$

where the $z_{i}$ are complex amplitudes and $\lambda, \epsilon$, and $b_{i}$ are real. An example of one of the family of such lattices is shown in Figure 4.1, where $\mathbf{K}_{\mathbf{i}_{\mathbf{h}}}$ is the mode with amplitude $z_{i}$. Different lattices correspond to different choices for $\theta$. In terms of the Faraday problem considered in this paper, these equations arise by representing the horizontal spatial dependence of the linear problem for the surface height $\zeta_{1}$ and the 


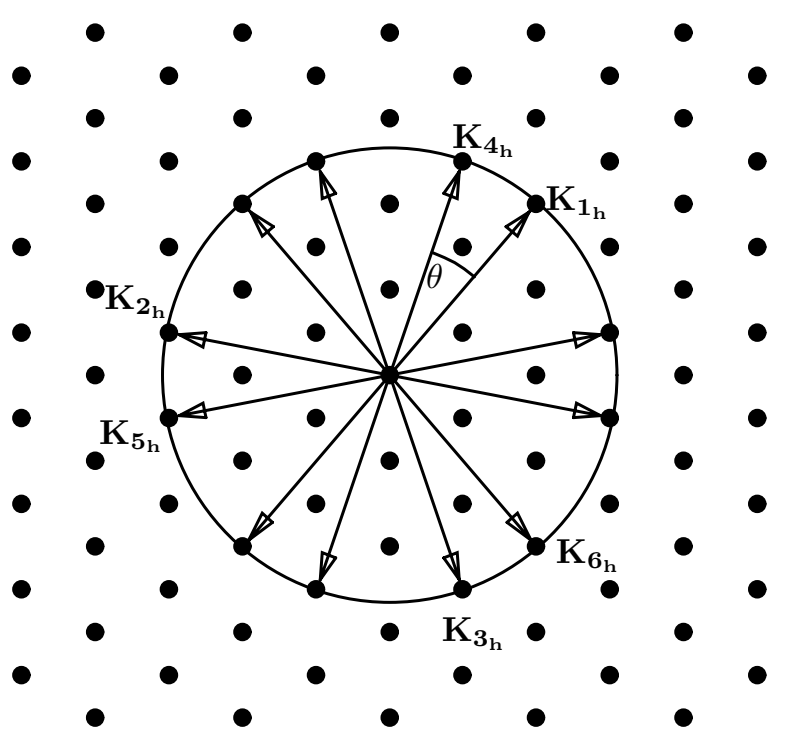

FIG. 4.1. Hexagonal lattice generated by 12 wavevectors on the critical circle.

vertical velocity $w_{1}$ as a sum of six modes where the $i$ th mode has amplitude $z_{i}$ and wavevector $K_{i_{h}}$.

For each hexagonal lattice, the equivariant branching lemma gives six patterns that bifurcate from the trivial state, and these are listed in Table 4.1 along with their branching equations and stability assignments. A further pattern has been found to exist and bifurcate from the trivial solution as discussed in [27]. We have not included this in Table 4.1 since its eigenvalues are indistinguishable from those for superhexagons at cubic order. We refer to both the superhexagons and the patterns discussed in [27] as superlattice patterns.

Similar results exist for families of square lattices.

Previously, the coefficients of the amplitude equations have been calculated for a long wavelength scalar partial differential equation describing a range of convection problems [28], for Turing patterns [29], and more recently for the Zhang-Viñals model of the Faraday problem [18]. These calculations allow inferences on the relative stability of different spatially periodic patterns to be made.

The cubic truncation of the amplitude equations (4.1) can be written in gradient form:

$$
\dot{z}_{i}=-\frac{\partial \mathcal{F}}{\partial \bar{z}_{i}},
$$

where the Lyapunov function is given by

$$
\begin{aligned}
\mathcal{F}= & -\sum_{i=1 . .6}\left[\lambda\left|z_{i}\right|^{2}-\frac{1}{2} b_{1}\left|z_{i}\right|^{4}\right] \\
& -\epsilon\left(z_{1} z_{2} z_{3}+z_{4} z_{5} z_{6}+\bar{z}_{1} \bar{z}_{2} \bar{z}_{3}+\bar{z}_{4} \bar{z}_{5} \bar{z}_{6}\right) \\
& -b_{2}\left(\left|z_{1}\right|^{2}\left|z_{2}\right|^{2}+\left|z_{1}\right|^{2}\left|z_{3}\right|^{2}+\left|z_{4}\right|^{2}\left|z_{5}\right|^{2}+\left|z_{4}\right|^{2}\left|z_{6}\right|^{2}+\left|z_{5}\right|^{2}\left|z_{6}\right|^{2}\right) \\
& -b_{4}\left(\left|z_{1}\right|^{2}\left|z_{4}\right|^{2}+\left|z_{2}\right|^{2}\left|z_{5}\right|^{2}+\left|z_{3}\right|^{2}\left|z_{6}\right|^{2}\right)-b_{5}\left(\left|z_{1}\right|^{2}\left|z_{5}\right|^{2}+\left|z_{2}\right|^{2}\left|z_{6}\right|^{2}+\left|z_{3}\right|^{2}\left|z_{4}\right|^{2}\right) \\
\text { (4.3) } & -b_{6}\left(\left|z_{1}\right|^{2}\left|z_{6}\right|^{2}+\left|z_{2}\right|^{2}\left|z_{4}\right|^{2}+\left|z_{3}\right|^{2}\left|z_{5}\right|^{2}\right) .
\end{aligned}
$$


TABLE 4.1

Branching equations and signs of eigenvalues for primary bifurcation branches on the hexagonal lattice; $\epsilon, b_{1}, \ldots, b_{6}$ are coefficients in the bifurcation equation (4.1).

\begin{tabular}{|c|c|}
\hline Branching equation & Signs of nonzero eigenvalues \\
\hline $\begin{array}{l}\text { Stripes (S) } \\
\mathbf{z}=\left(A_{S}, 0,0,0,0,0\right) \\
0=\lambda A_{S}+b_{1} A_{S}^{3}+\mathcal{O}\left(A_{S}^{5}\right)\end{array}$ & $\begin{array}{l}\operatorname{sgn}\left(b_{1}\right), \quad \operatorname{sgn}\left(\tilde{\epsilon} A_{S}+\left(b_{2}-b_{1}\right) A_{S}^{2}\right), \\
\operatorname{sgn}\left(-\tilde{\epsilon} A_{S}+\left(b_{2}-b_{1}\right) A_{S}^{2}\right), \\
\operatorname{sgn}\left(b_{4}-b_{1}\right), \quad \operatorname{sgn}\left(b_{5}-b_{1}\right), \quad \operatorname{sgn}\left(b_{6}-b_{1}\right) .\end{array}$ \\
\hline $\begin{array}{l}\text { Simple hexagons }\left(\mathrm{H}^{ \pm}\right) \\
\begin{aligned} & \mathbf{z}=\left(A_{H}, A_{H}, A_{H}, 0,0,0\right) \\
& 0=\lambda A_{H}+\tilde{\epsilon} A_{H}^{2} \\
& \quad+\left(b_{1}+2 b_{2}\right) A_{H}^{3}+\mathcal{O}\left(A_{H}^{4}\right)\end{aligned}\end{array}$ & $\begin{array}{l}\operatorname{sgn}\left(\tilde{\epsilon} A_{H}+2\left(b_{1}+2 b_{2}\right) A_{H}^{2}\right) \\
\operatorname{sgn}\left(-\tilde{\epsilon} A_{H}+\left(b_{1}-b_{2}\right) A_{H}^{2}\right) \\
\operatorname{sgn}\left(-\tilde{\epsilon} A_{H}+\left(b_{4}+b_{5}+b_{6}-b_{1}-2 b_{2}\right) A_{H}^{2}\right) \\
\operatorname{sgn}\left(-\tilde{\epsilon} A_{H}+\mathcal{O}\left(A_{H}^{3}\right)\right)\end{array}$ \\
\hline $\begin{array}{l}\text { Rectangles }\left(\mathrm{R}_{h 1, m, n}\right) \\
\mathbf{z}=\left(A_{R}, 0,0, A_{R}, 0,0\right) \\
0=\lambda A_{R}+\left(b_{1}+b_{4}\right) A_{R}^{3}+\mathcal{O}\left(A_{R}^{5}\right)\end{array}$ & $\begin{array}{l}\operatorname{sgn}\left(b_{1}+b_{4}\right), \quad \operatorname{sgn}\left(b_{1}-b_{4}\right) \\
\operatorname{sgn}\left(\mu_{1}\right), \quad \operatorname{sgn}\left(\mu_{2}\right), \quad \text { where } \\
\mu_{1}+\mu_{2}=\left(-2 b_{1}-2 b_{4}+2 b_{2}+b_{5}+b_{6}\right) A_{R}^{2} \\
\mu_{1} \mu_{2}=-\tilde{\epsilon}^{2} A_{R}^{2}+\left(b_{1}+b_{4}-b_{2}-b_{5}\right) \\
\quad\left(b_{1}+b_{4}-b_{2}-b_{6}\right) A_{R}^{4}\end{array}$ \\
\hline $\begin{array}{l}\text { Rectangles }\left(\mathrm{R}_{h 2, m, n}\right) \\
\mathbf{z}=\left(A_{R}, 0,0,0, A_{R}, 0\right) \\
0=\lambda A_{R}+\left(b_{1}+b_{5}\right) A_{R}^{3}+\mathcal{O}\left(A_{R}^{5}\right)\end{array}$ & $\begin{array}{l}\operatorname{sgn}\left(b_{1}+b_{5}\right), \quad \operatorname{sgn}\left(b_{1}-b_{5}\right) \\
\operatorname{sgn}\left(\mu_{1}\right), \quad \operatorname{sgn}\left(\mu_{2}\right), \quad \text { where } \\
\mu_{1}+\mu_{2}=\left(-2 b_{1}-2 b_{5}+2 b_{2}+b_{4}+b_{6}\right) A_{R}^{2} \\
\mu_{1} \mu_{2}=-\tilde{\epsilon}^{2} A_{R}^{2}+\left(b_{1}+b_{5}-b_{2}-b_{4}\right) \\
\quad\left(b_{1}+b_{5}-b_{2}-b_{6}\right) A_{R}^{4}\end{array}$ \\
\hline $\begin{array}{l}\text { Rectangles }\left(\mathrm{R}_{h 3, m, n}\right) \\
\mathbf{z}=\left(A_{R}, 0,0,0,0, A_{R}\right) \\
0=\lambda A_{R}+\left(b_{1}+b_{6}\right) A_{R}^{3}+\mathcal{O}\left(A_{R}^{5}\right)\end{array}$ & $\begin{array}{l}\operatorname{sgn}\left(b_{1}+b_{6}\right), \quad \operatorname{sgn}\left(b_{1}-b_{6}\right) \\
\operatorname{sgn}\left(\mu_{1}\right), \quad \operatorname{sgn}\left(\mu_{2}\right), \quad \text { where } \\
\mu_{1}+\mu_{2}=\left(-2 b_{1}-2 b_{6}+2 b_{2}+b_{4}+b_{5}\right) A_{R}^{2} \\
\mu_{1} \mu_{2}=-\tilde{\epsilon}^{2} A_{R}^{2}+\left(b_{1}+b_{6}-b_{2}-b_{4}\right) \\
\quad\left(b_{1}+b_{6}-b_{2}-b_{5}\right) A_{R}^{4}\end{array}$ \\
\hline $\begin{array}{l}\text { Superhexagons }\left(\mathrm{SH}_{m, n}^{ \pm}\right) \\
\quad \begin{array}{l}\mathbf{z}= \\
\quad A_{S H}, A_{S H}, A_{S H} \\
\left.\quad A_{S H}, A_{S H}, A_{S H}\right) \\
0=\lambda A_{S H}+\tilde{\epsilon} A_{S H}^{2}+\left(b_{1}+2 b_{2}\right) A_{S H}^{3} \\
\quad+\left(b_{4}+b_{5}+b_{6}\right) A_{S H}^{3}+\mathcal{O}\left(A_{S H}^{4}\right)\end{array}\end{array}$ & $\begin{array}{l}\operatorname{sgn}\left(\tilde{\epsilon} A_{S H}+2\left(b_{1}+2 b_{2}+b_{4}+b_{5}+b_{6}\right) A_{S H}^{2}\right), \\
\operatorname{sgn}\left(\tilde{\epsilon} A_{S H}+2\left(b_{1}+2 b_{2}-b_{4}-b_{5}-b_{6}\right) A_{S H}^{2}\right), \\
\operatorname{sgn}\left(-\tilde{\epsilon} A_{S H}+\mathcal{O}\left(A_{S H}^{3}\right)\right), \\
\operatorname{sgn}\left(-\tilde{\epsilon} A_{S H}+\mathcal{O}\left(A_{S H}^{3}\right)\right),{ }^{*} \\
\operatorname{sgn}\left(\mu_{1}\right), \quad \operatorname{sgn}\left(\mu_{2}\right), \quad \text { where } \\
\mu_{1}+\mu_{2}=-4 \tilde{\epsilon} A_{S H}+4\left(b_{1}-b_{2}\right) A_{S H}^{2}, \\
\mu_{1} \mu_{2}=4\left(\tilde{\epsilon} A_{S H}-\left(b_{1}-b_{2}\right) A_{S H}^{2}\right)^{2} \\
\quad-2\left(\left(b_{4}-b_{5}\right)^{2}+\left(b_{4}-b_{6}\right)^{2}\right. \\
\left.\left.\quad+\left(b_{5}-b_{6}\right)^{2}\right)\right) A_{S H}^{4}, \\
\operatorname{sgn}\left(\mu_{0}\right), \text { where } \mu_{0}=\mathcal{O}\left(A_{S H}^{2(m-1)}\right) .\end{array}$ \\
\hline
\end{tabular}

*These two eigenvalues differ at $\mathcal{O}\left(A_{S H}^{3}\right)$.

The different planforms then correspond to minima of the Lyapunov functional, and an "energy" for each state may be computed. In Table 4.2, we list the different planforms and the corresponding value of the Lyapunov function (4.3). The information given by the eigenvalues of the amplitude equations given in Table 4.1 and that given by the energy of the different states as given in Table 4.2 is complementary. Below we calculate the coefficients for the amplitude equations (4.1) from the full Navier-Stokes equation formulation of the Faraday problem. We then calculate the eigenvalues to examine relative stability. For those states that are relatively stable, we calculate the value of the Lyapunov function to find which have the lowest energy.

The coefficients of the amplitude equations are found by focusing on three calculations: one for stripes, one for rectangular patterns, and one for hexagons; these correspond to considering the three subspaces, $\mathbf{z}=\left(A_{S}, 0,0,0,0,0\right), \mathbf{z}=\left(A_{R}, 0,0, A_{R}, 0,0\right)$, 
TABLE 4.2

Value of the Lyapunov function $\mathcal{F}$ for each of the primary bifurcation branches on the hexagonal lattice; $\epsilon, b_{1}, \ldots, b_{6}$ are coefficients in the bifurcation equation (4.1). Only one of the rectangular states has been included: the other two may be obtained by cyclic permutation.

\begin{tabular}{|l|l|}
\hline Planform & $\mathcal{F}$ \\
\hline $\begin{array}{l}\text { Stripes }(\mathrm{S}) \\
\mathbf{z}=\left(A_{S}, 0,0,0,0,0\right)\end{array}$ & $\frac{\lambda^{2}}{b_{1}}$ \\
\hline $\begin{array}{l}\text { Simple hexagons }\left(\mathrm{H}^{ \pm}\right) \\
\mathbf{z}=\left(A_{H}, A_{H}, A_{H}, 0,0,0\right)\end{array}$ & $\begin{array}{l}-\left(3 \lambda A_{H}^{2}+2 \epsilon A_{H}^{3}+\frac{3}{2}\left(b_{1}+2 b_{2}\right) A_{H}^{4}\right), \\
\text { where } 0=\lambda+\epsilon A_{H}+\left(b_{1}+2 b_{2}\right) A_{H}^{2}\end{array}$ \\
\hline $\begin{array}{l}\text { Rectangles }\left(\mathrm{R}_{h 1, m, n}\right) \\
\mathbf{z}=\left(A_{R}, 0,0, A_{R}, 0,0\right)\end{array}$ & $\frac{\lambda^{2}}{\left(b_{1}+b_{4}\right)}$ \\
\hline $\begin{array}{l}\text { Superhexagons }\left(\mathrm{SH}_{m, n}^{ \pm}\right) \\
\mathbf{z}=\left(A_{S H}, A_{S H}, A_{S H}, A_{S H}, A_{S H}, A_{S H}\right)\end{array}$ & \begin{tabular}{l} 
where $0=\lambda+\tilde{\epsilon} A_{S H}+\left(b_{1}+2 b_{2}+b_{4}+b_{5}+b_{6}\right) A_{S H}^{2}$ \\
\hline
\end{tabular} \\
\hline
\end{tabular}

and $\mathbf{z}=\left(A_{H}, A_{H}, A_{H}, 0,0,0\right)$, respectively. Focusing on a particular pattern means that we make an assumption about the particular form of the horizontal behavior of the fluid variables. We can use this to reformulate the weakly nonlinear analysis in section 3 that is in terms of functions of $x, y$, and $z$ to a simpler set of problems for sets of functions that depend only on $z$. It is this reformulation of the problem that is carried out in this section for each of the stripes, rectangles, and hexagons. Since stripes and rectangles arise through a symmetry-breaking bifurcation, there are no quadratic terms in the amplitude equations for these patterns. A result of this is that the solvability condition at second order necessarily leads to $a_{1}=0$. This fact can be included from the beginning of the analysis, and then we need only scale on two timescales; that is, we let $t=\tau+T / \varepsilon^{2}$ so that $\partial_{t}=\partial_{\tau}+\varepsilon^{2} \partial_{T}$. (The more general formulation on three timescales is needed for hexagons.)

For stripes, we consider a solution to the first order problem given in section 3.1 of the form

$$
\begin{gathered}
w_{1}(x, z, \tau, T)=A_{S}(T)\left(e^{i k x}+e^{-i k x}\right) \sum_{n} W_{1, n}(z) e^{i(n \omega+\alpha) \tau}, \\
\zeta_{1}(x, \tau, T)=A_{S}(T)\left(e^{i k x}+e^{-i k x}\right) \sum_{n} Z_{1, n} e^{i(n \omega+\alpha) \tau},
\end{gathered}
$$

and for rectangular patterns, we consider

$$
\begin{gathered}
w_{1}(x, y, z, \tau, T)=A_{R}(T)\left[e^{i k x}+e^{i k(c x+s y)}+c . c .\right] \sum_{n} W_{1, n}(z) e^{i(n \omega+\alpha) \tau}, \\
\zeta_{1}(x, y, \tau, T)=A_{R}(T)\left[e^{i k x}+e^{i k(c x+s y)}+c . c .\right] \sum_{n} Z_{1, n} e^{i(n \omega+\alpha) \tau},
\end{gathered}
$$

where $s=\sin \theta$ and $c=\cos \theta$ and $\theta$ is the angle between the wavevectors that make up the rectangular pattern. Here $k$ is the wavenumber of the pattern, and a Floquet expansion in the basic frequency $\omega$ has been used as in [14]. When $\alpha=0$, the expansion gives a harmonic solution, and when $\alpha=\omega / 2$, the expansion gives a subharmonic 
solution. Here and below we sum from $n=-\infty$ to $n=+\infty$. Consequently, since both the $w_{1}$ and $\zeta_{1}$ are real, we have in addition

$$
\begin{aligned}
& W_{1, n}=\bar{W}_{1,-n}, \quad \alpha=0, \\
& W_{1, n}=\bar{W}_{1,-n-1}, \quad \alpha=\omega / 2 .
\end{aligned}
$$

Although we do not list them, there are analogous reality conditions for the velocity components and surface height at each order. Similar choices for the expansion are made for the behavior of the horizontal velocity components $u_{1}$ and $v_{1}$, and these are listed in Appendix A. Since the results for stripes can be obtained by setting $\theta=0$ and careful consideration of some factors of two, in what follows we include stripes as a special case in our formulation of the problem for rectangles. In order to proceed, the general form (4.4) for the pattern is substituted into the first order problem given in section 3.1. The result is a homogeneous fourth order linear differential equation for the vertical dependence of the vertical velocity component $W_{1, n}(z)$ along with the appropriate boundary conditions at $z=0$ and $z=-h / l$. This is given in section 4.1.

At second order, as for the first order problem, assuming that we are interested in particular patterns means that we know the form for the horizontal behavior of the fluid. Specifically, we take the general form of the second order solution for rectangles as

$$
\begin{aligned}
w_{2}(x, y, z, \tau, T)= & A_{R}^{2}(T)\left[e^{2 i k x}+e^{2 i k(c x+s y)}+c . c .\right] \sum_{n} W_{2,1, n}(z) e^{i(n \omega+2 \alpha) \tau} \\
& +A_{R}^{2}(T)\left[e^{i k[(1+c) x+s y]}+c . c .\right] \sum_{n} W_{2,2, n}(z) e^{i(n \omega+2 \alpha) \tau} \\
& +A_{R}^{2}(T)\left[e^{i k[(1-c) x-s y]}+c . c .\right] \sum_{n} W_{2,3, n}(z) e^{i(n \omega+2 \alpha) \tau}, \\
\zeta_{2}(x, y, \tau, T)= & A_{R}^{2}(T)\left[e^{2 i k x}+e^{2 i k(c x+s y)}+c . c .\right] \sum_{n} Z_{2,1, n} e^{i(n \omega+2 \alpha) \tau} \\
& +A_{R}^{2}(T)\left[e^{i k[(1+c) x+s y]}+c . c .\right] \sum_{n} Z_{2,2, n} e^{i(n \omega+2 \alpha) \tau} \\
& +A_{R}^{2}(T)\left[e^{i k[(1-c) x-s y]}+\text { c.c. }\right] \sum_{n} Z_{2,3, n} e^{i(n \omega+2 \alpha) \tau} .
\end{aligned}
$$

The forms that are taken for the velocity components $u_{2}$ and $v_{2}$ are given in Appen$\operatorname{dix} \mathrm{A}$. The expressions for the velocity components are substituted into the equations and boundary conditions at second order given in section 3.2, and this leads to an inhomogeneous fourth order ordinary differential equation for $W_{2, i, n}(z)$ along with boundary conditions. These are given in section 4.2.

As discussed above, if we take $a_{1}=0$, then there is no solvability condition at second order for rectangles. However, at third order there is a solvability condition. In order to derive this, the general form for the adjoint problem is needed. In section 4.3, we give the formulation for the adjoint problem, derived from the adjoint problem given in (3.8) along with the assumption that patterns to the adjoint problem take the general form

$$
\begin{aligned}
w^{*}(x, z, \tau, T) & =A^{*}(T)\left(e^{i k x}+e^{-i k x}\right) \sum_{n} W_{n}^{*}(z) e^{i(n \omega+\alpha) \tau}, \\
\zeta^{*}(x, \tau, T) & =A^{*}(T)\left(e^{i k x}+e^{-i k x}\right) \sum_{n} Z_{n}^{*} e^{i(n \omega+\alpha) \tau} .
\end{aligned}
$$


This then allows us to formulate the solvability condition at third order in section 4.4. The result is an amplitude equation for rectangles whose coefficients may be determined from $W_{1, n}(z), Z_{1, n}, W_{2, i, n}(z), Z_{2, i, n}$ and their derivatives along with the adjoint eigenfunctions $W_{1, n}^{*}(z)$ and $Z_{1, n}^{*}$ and their derivatives.

In the case of hexagons, we consider a first order solution of the form

$$
\begin{aligned}
& w_{1}\left(x, y, z, \tau, T_{1}, T_{2}\right) \\
& \quad=A_{H}\left(T_{1}, T_{2}\right)\left[e^{i k x}+e^{i k(-x+\sqrt{3} y) / 2}+e^{i k(-x-\sqrt{3} y) / 2}+\text { c.c. }\right] \sum_{n} W_{1, n}(z) e^{i(n \omega+\alpha) \tau},
\end{aligned}
$$

$$
\begin{aligned}
& \zeta_{1}\left(x, y, z, \tau, T_{1}, T_{2}\right) \\
& \quad=A_{H}\left(T_{1}, T_{2}\right)\left[e^{i k x}+e^{i k(-x+\sqrt{3} y) / 2}+e^{i k(-x-\sqrt{3} y) / 2}+\text { c.c. }\right] \sum_{n} Z_{1, n} e^{i(n \omega+\alpha) \tau}
\end{aligned}
$$

with similar choices for $u_{1}$ and $v_{1}$ that are listed in Appendix A. This leads to the same first order problem as for stripes and rectangles, as given in section 4.1 below.

At second order hexagons differ from rectangles. Generically, in problems that have $\mathrm{E}(2)$ symmetry, hexagons arise in a transcritical bifurcation and are necessarily locally unstable. In the weakly nonlinear analysis, this appears as a quadratic amplitude equation that results from the solvability condition for the second order problem. Two cases of interest arise that can result in stable hexagonal solutions: first, when there is an extra symmetry in the problem that removes the quadratic term, and second, when the coefficient of the quadratic term is sufficiently small so that the quadratic terms may formally be included at cubic order [30]. The Faraday problem is an example of a system that has $\mathrm{E}(2)$ symmetry. As we shall see below, for some values of the parameter $\chi$ in the drive (2.3), the response is subharmonic, and for some values it is harmonic. When the response is subharmonic, then there is an extra time symmetry in the problem and there are no quadratic terms in the amplitude equations. When $\chi=0$, the response is harmonic, but since there is no $M_{2}$ component in the drive, there is again an extra symmetry in the problem, and again there are no quadratic terms in the amplitude equations. However, as the parameter $\chi$ is increased from 0 , this extra symmetry is broken, and there is a gradual increase from zero in the size of the coefficient of the quadratic term. There is therefore at least some range in parameter space where it is reasonable to include the quadratic terms at cubic order. The way we proceed with the hexagon calculation is therefore as follows. First, we consider three timescales and formulate the solvability condition at second order. This is done using the solvability condition given in (3.9) and the specific form for the hexagonal pattern at first order (4.7); the result is given in section 4.5. This gives us a quadratic amplitude equation and enables us to compute the size of the quadratic term.

Next, we make the assumption that the coefficient of the quadratic term is either zero or sufficiently small $(O(\varepsilon))$ so that we may formally include the terms at cubic order. We therefore set $a_{1}=0$, rescale the problem on two timescales, and formulate the problem for the solution at second order by taking as a general form for the second order problem

$$
\begin{gathered}
w_{2}(x, y, z, \tau, T)=A_{H}^{2}\left[e^{2 i k x}+e^{i k(-x+\sqrt{3} y)}+e^{i k(-x-\sqrt{3} y)}+\text { c.c. }\right] \sum_{n} W_{2,1, n}(z) e^{i(n \omega+2 \alpha) \tau} \\
+A_{H}^{2}\left[e^{i k \sqrt{3} y}+e^{i k(3 x+\sqrt{3} y) / 2}+e^{i k(3 x-\sqrt{3} y) / 2}+\text { c.c. }\right] \sum_{n} W_{2,2, n}(z) e^{i(n \omega+2 \alpha) \tau}
\end{gathered}
$$


$\zeta_{2}(x, y, \tau, T)=A_{H}^{2}\left[e^{2 i k x}+e^{i k(-x+\sqrt{3} y)}+e^{i k(-x-\sqrt{3} y)}+\right.$ c.c. $] \sum_{n} Z_{2,1, n} e^{i(n \omega+2 \alpha) \tau}$

$$
+A_{H}^{2}\left[e^{i k \sqrt{3} y}+e^{i k(3 x+\sqrt{3} y) / 2}+e^{i k(3 x-\sqrt{3} y) / 2}+\text { c.c. }\right] \sum_{n} Z_{2,2, n} e^{i(n \omega+2 \alpha) \tau} .
$$

Expressions for $u_{2}$ and $v_{2}$ are listed in Appendix A. Substitution of these expressions into the second order equation and boundary conditions given in section 3.2 leads to an inhomogeneous fourth order ordinary differential equation for $W_{2, i, n}$ along with boundary conditions. These are given in section 4.6. Finally, including the quadratic terms at cubic order, we formulate the solvability condition for the third order hexagonal problem in section 4.7.

4.1. The linear problem. The linear problem is the same for all periodic patterns and is given by

$$
\left[i(n \omega+\alpha)-C\left(D^{2}-k^{2}\right)\right]\left(D^{2}-k^{2}\right) W_{1, n}(z)=0,
$$

where $D$ indicates the derivative with respect to $z$, with boundary conditions

$$
W_{1, n}=D W_{1, n}=0,
$$

on $z=-h / l$, and on $z=0$,

$$
\begin{aligned}
i(n \omega+\alpha) Z_{1, n}-W_{1, n} & =0 \\
\left(D^{2}+k^{2}\right) W_{1, n} & =0 \\
\left(i(n \omega+\alpha)+3 C k^{2}\right) D W_{1, n}-C D^{3} W_{1, n}+k^{2}\left(B k^{2}+1\right) Z_{1, n} & =-\frac{1}{2} a_{0} k^{2} Z_{1, f, n} .
\end{aligned}
$$

For a single frequency of excitation,

$$
Z_{1, f, n}=Z_{1, n-1}+Z_{1, n+1}
$$

and for two frequencies,

$$
Z_{1, f, n}=\cos (\chi)\left(Z_{1, n-M_{1}}+Z_{1, n+M_{1}}\right)+\sin (\chi)\left(e^{i \phi} Z_{1, n-M_{2}}+e^{-i \phi} Z_{1, n+M_{2}}\right) .
$$

These equations are supplemented by the reality conditions

$$
W_{1,-n}(0)=\bar{W}_{1, n}(0), \quad Z_{1,-n}=\bar{Z}_{1, n}
$$

for harmonic modes and

$$
W_{1,-n}(0)=\bar{W}_{1, n-1}(0), \quad Z_{1,-n}=\bar{Z}_{1, n-1}
$$

for subharmonic modes, where the bar indicates complex conjugation.

4.2. Second order problem for rectangles and stripes. The functions $W_{2, i, n}$ and $Z_{2, i, n}, i=1,2,3$, can all be found from the same system of equations with different choices made for the parameters $\theta$ and $d$. For $i=1$, we take $\theta=0$ and $d=1 / 2$ : this would be the same as solving for stripes. For $i=2$, we take $\theta=\tilde{\theta}$ and $d=1$. For $i=3$, we take $\theta=\pi+\tilde{\theta}$ and $d=1$, where $\tilde{\theta} \in(0, \pi / 2]$. The solutions $W_{2, i, n}$ satisfy

$$
\begin{gathered}
{\left[i(n \omega+2 \alpha)-C\left(D^{2}-2 k^{2}(1+c)\right)\right]\left(D^{2}-2 k^{2}(1+c)\right) W_{2, i, n}(z)} \\
=d \sum_{l+m=n}\left[4 k^{2} s^{2} D W_{1, l}(z) W_{1, m}(z)-2(1+c) D^{3} W_{1, l}(z) W_{1, m}(z)\right. \\
\left.+2\left(-1+c+2 c^{2}\right) D^{2} W_{1, l}(z) D W_{1, m}(z)\right]
\end{gathered}
$$


at $z=-h / l$,

$$
W_{2, i, n}=D W_{2, i, n}=0,
$$

while at $z=0$ we have the kinematic condition and the tangential stress condition,

$$
\begin{aligned}
i(n \omega+2 \alpha) Z_{2, i, n}-W_{2, i, n} & =2 d(1+c) \sum_{l+m=n} D W_{1, l} Z_{1, m}, \\
\left(D^{2}+2 k^{2}(1+c)\right) W_{2, i, n}=-d \sum_{l+m=n}[ & 2(1+c) Z_{1, l} D^{3} W_{1, m} \\
+ & \left.+2(3+2 c)(1+c) k^{2} Z_{1, l} D W_{1, m}\right],
\end{aligned}
$$

and the normal stress condition,

$$
\begin{aligned}
& {\left[i(n \omega+2 \alpha)+6(1+c) C k^{2}\right] D W_{2, i, n}-C D^{3} W_{2, i, n}} \\
& (4.20) \quad+2 k^{2}(1+c)\left(2 B(1+c) k^{2}+1\right) Z_{2, i, n}=S_{1, i, n}-a_{0} k^{2}(1+c) Z_{2, f, i, n},
\end{aligned}
$$

where

$$
\begin{aligned}
S_{1, i, n}= & d \sum_{l+m=n}\left[2 c(1+c) D W_{1, l} D W_{1, m}\right. \\
& \left.+4(1+c) k^{2} Z_{1, l} D P_{1, m}-8(1+c) C k^{2} Z_{1, l} D^{2} W_{1, m}-2(1+c) W_{1, l} D^{2} W_{1, m}\right] .
\end{aligned}
$$

For a single frequency,

$$
Z_{2, f, i, n}=Z_{2, i, n-1}+Z_{2, i, n+1}
$$

and for two frequencies,

$$
Z_{2, f, i, n}=\cos (\chi)\left(Z_{2, i, n-M_{1}}+Z_{2, i, n+M_{1}}\right)+\sin (\chi)\left(e^{i \phi} Z_{2, i, n-M_{2}}+e^{-i \phi} Z_{2, i, n+M_{2}}\right) .
$$

Note that if there is no extra symmetry that suppresses it, then the resonant triad interaction at second order causes this calculation to blow up at $\theta=\pi / 3$.

4.3. Linear adjoint problem. The adjoint problem is

$$
\left[i(n \omega+\alpha)+C\left(D^{2}-k^{2}\right)\right]\left(D^{2}-k^{2}\right) W_{n}^{*}(z)=0,
$$

with boundary conditions

$$
W_{n}^{*}=D W_{n}^{*}=0,
$$

on $z=-h / l$, and on $z=0$,

$$
\begin{aligned}
{\left[i(n \omega+\alpha)-3 C k^{2}\right] D W_{n}^{*}+C D^{3} W_{n}^{*} } & =Z_{n}^{*} \\
\left(D^{2}+k^{2}\right) W_{n}^{*} & =0 \\
i(n \omega+\alpha) Z_{n}^{*}+k^{2}\left(B k^{2}+1\right) W_{n}^{*} & =-\frac{1}{2} a_{0} k^{2} W_{f, n}^{*} .
\end{aligned}
$$

For a single frequency,

$$
W_{f, n}^{*}=W_{n-1}^{*}+W_{n+1}^{*},
$$

and for two frequencies,

$$
W_{f, n}^{*}=\cos (\chi)\left(W_{n-M_{1}}^{*}+W_{n+M_{1}}^{*}\right)+\sin (\chi)\left(e^{i \phi} W_{n-M_{2}}^{*}+e^{-i \phi} W_{n+M_{2}}^{*}\right) .
$$


4.4. Solvability condition for rectangles and stripes. The solvability condition is

$$
\delta \frac{d A}{d T}=a_{2} \beta A+\gamma A^{3},
$$

where $A \equiv A_{S}$ in Table 4.1 for stripes and $A \equiv A_{R}$ in Table 4.1 for rectangles and

$$
\begin{aligned}
\delta=\sum_{l, m}(1) & {\left[-2 Z_{l}^{*} Z_{1, m}+2 W_{l}^{*}(0) D W_{1, m}(0)\right.} \\
& \left.+2 \int_{-h / l}^{0} W_{l}^{*}(z)\left(-D^{2} W_{1, m}(z)+k^{2} W_{1, m}(z)\right) d z\right] .
\end{aligned}
$$

For one frequency,

$$
\beta=-k^{2}\left[\sum_{l, m}^{(4)} W_{l}^{*}(0) Z_{1, m}+\sum_{l, m}^{(5)} W_{l}^{*}(0) Z_{1, m}\right],
$$

and for two frequencies,

$$
\begin{aligned}
\beta=-k^{2}[ & \cos (\chi)\left(\sum_{l, m}^{(6)} W_{l}^{*}(0) Z_{1, m}+\sum_{l, m}^{(7)} W_{l}^{*}(0) Z_{1, m}\right) \\
& \left.+\sin (\chi)\left(\sum_{l, m}^{(8)} W_{l}^{*}(0) e^{i \psi} Z_{1, m}+\sum_{l, m}^{(9)} W_{l}^{*}(0) e^{-i \psi} Z_{1, m}\right)\right] .
\end{aligned}
$$

The coefficient $\gamma$ is given by the sum of three separate components corresponding to contributions from each of $W_{2, i, n}$ and $Z_{2, i, n}$. They may each be calculated from a single function $\gamma_{i}\left(\theta_{s}, d\right)$ by taking each of the three functions in turn and different $\theta_{s}$ and $d$. Specifically,

$$
\gamma\left(\theta_{s}, d\right)=\gamma_{1}\left(0, \frac{1}{2}\right)+\gamma_{2}\left(\theta_{s}, 1\right)+\gamma_{3}\left(\pi+\theta_{s}, 1\right) .
$$

The function $\gamma_{i}$ is given in Appendix B.

4.5. Second order solvability condition for hexagons. The second order solvability condition for hexagons leads to the amplitude equation

$$
\delta \frac{d A_{H}}{d T_{1}}=a_{1} \beta A_{H}+\gamma_{2} A_{H}^{2},
$$

and $\gamma_{2}$ is given by

$$
\begin{aligned}
\gamma_{2}= & -2 \sum_{l, m, n}^{(2)} Z_{l}^{*} Z_{1, m} D W_{1, n}(0) \\
& -\sum_{l, m, n}^{(2)} W_{l}^{*}(0)\left[D W_{1, m}(0) D W_{1, n}(0)+8 C k^{2} Z_{1, m} D^{2} W_{1, n}(0)\right] \\
& +\sum_{l, m, n}^{(2)} W_{l}^{*}(0)\left[4 k^{2} Z_{1, m} D P_{1, n}(0)-2 W_{1, m}(0) D^{2} W_{1, n}(0)\right]
\end{aligned}
$$




$$
\begin{gathered}
-C \sum_{l, m, n}^{(2)} D W_{l}^{*}(0) Z_{1, m}\left[2 D^{3} W_{1, n}(0)+4 k^{2} D W_{1, n}(0)\right] \\
-\sum_{l, m, n}^{(2)} \int_{-h / l}^{0} W_{l}^{*}(z)\left[W_{1, m}(z)\left(6 k^{2} D W_{1, n}(z)-2 D^{3} W_{1, n}(z)\right)\right. \\
\left.-4 D W_{1, m}(z) D^{2} W_{1, n}(z)\right] d z .
\end{gathered}
$$

4.6. Second order problem for hexagons. The second order problem for hexagons consists of $W_{2,1, n}(z)=W_{2, n}(z)$, which solves the same problem as that obtained for stripes, and $W_{2,2, n}$, which satisfies

$$
\begin{aligned}
{[i(n \omega+2 \alpha)} & \left.-C\left(D^{2}-3 k^{2}\right)\right]\left(D^{2}-3 k^{2}\right) W_{2,2, n}(z) \\
& =\sum_{l+m=n}\left(3 k^{2} W_{1, l}(z) D W_{1, m}(z)-3 D^{3} W_{1, l}(z) W_{1, m}(z)\right) .
\end{aligned}
$$

At $z=-h / l$ we have $W_{2,2, n}=D W_{2,2, n}=0$, while at $z=0$ we have the kinematic condition and the tangential stress conditions, namely,

$$
\begin{aligned}
i(n \omega+2 \alpha) Z_{2,2, n}-W_{2,2, n} & =3 \sum_{l+m=n} Z_{1, l} D W_{1, m}, \\
\left(3 k^{2}+D^{2}\right) W_{2,2, n} & =-\sum_{l+m=n}\left[3 Z_{1, l} D^{3} W_{1, m}+12 k^{2} Z_{1, l} D W_{1, m}\right],
\end{aligned}
$$

and the normal stress condition,

$$
\begin{aligned}
& \left(i(n \omega+2 \alpha)+9 C k^{2}\right) D W_{2,2, n}-C D^{3} W_{2,2, n}+3 k^{2}\left(3 B k^{2}+1\right) Z_{2,2, n} \\
& \quad=S_{1, h, n}-\frac{3}{2} a_{0} k^{2} Z_{2, f, n},
\end{aligned}
$$

where

$$
\begin{array}{r}
S_{1, h, n}=\sum_{l+m=n}\left(\frac{3}{2} D W_{1, l} D W_{1, m}-12 C k^{2} Z_{1, l} D^{2} W_{1, m}\right. \\
\left.+6 k^{2} Z_{1, l} D P_{1, m}-3 W_{1, l} D^{2} W_{1, m}\right) .
\end{array}
$$

For a single frequency of excitation,

$$
Z_{2, f, n}=Z_{2,2, n-1}+Z_{2,2, n+1},
$$

and for two frequencies,

$$
Z_{2, f, n}=\cos (\chi)\left(Z_{2,2, n-M_{1}}+Z_{2,2, n+M_{1}}\right)+\sin (\chi)\left(e^{i \phi} Z_{2,2, n-M_{2}}+e^{-i \phi} Z_{2,2, n+M_{2}}\right) .
$$

4.7. Solvability condition for hexagons at third order. The solvability condition at third order problem takes the form

$$
\delta \frac{d A_{H}}{d T}=a_{2} \beta A_{H}+\gamma_{2} A_{H}^{2}+\left(\gamma_{1}+\gamma_{3}\right) A_{H}^{3},
$$

where $\delta, \beta$, and $\gamma_{1}$ are the same as for stripes, $\gamma_{2}$ is given by (4.31), and $\gamma_{3}$ is given in Appendix C. 
5. Calculation of the coefficients of the amplitude equations. In section 4, we formulated a hierarchy of problems for the $z$ dependence of the fluid parameters. In this section, we outline how we solve this sequence of problems and calculate the coefficients of the amplitude equations. We focus on the harmonic case, $\alpha=0$, but the calculations for the subharmonic case are similar. We also note that using the transformation $\omega \rightarrow \omega / 2, M_{1} \rightarrow 2 M_{1}, M_{2} \rightarrow 2 M_{2}$, the subharmonic case can be incorporated into the formulation for the harmonic problem. As explained in section 5.1, the calculations are substantially simpler in the specific case of an infinite layer, and while we have solved the linear problem for both the finite and infinite cases, we have calculated the coefficients of the amplitude equations only for infinite depth. We justify why this is a reasonable approximation for the specific fluid parameter choices we use when we discuss the results in section 6 .

5.1. Linear problem. The general solution for $W_{1, n}(z)$ that satisfies (4.9) is

$$
\begin{aligned}
& W_{1,0}(z)=\left(a_{1,0}+z c_{1,0}\right) e^{k z}+\left(b_{1,0}+z d_{1,0}\right) e^{-k z}, \\
& W_{1, n}(z)=a_{1, n} e^{k z}+b_{1, n} e^{-k z}+c_{1, n} e^{q_{1, n} z}+d_{1, n} e^{-q_{1, n} z}, \quad n \neq 0,
\end{aligned}
$$

where

$$
q_{1, n}^{2}=\frac{i n \omega}{C}+k^{2} .
$$

Applying the boundary conditions allows values for the coefficients $a_{1, n}, b_{1, n}, c_{1, n}$, and $d_{1, n}$ to be found. However, the subsequent analysis is substantially easier in the particular case of an infinite layer, where the lower boundary conditions are replaced with the requirement that the solution be bounded as $z \rightarrow-\infty$. In this case, $b_{1, n}=$ $d_{1, n}=0$, and it is this case we discuss below.

In order to find $a_{1, n}$ and $c_{1, n}$, they are expressed first in terms of $Z_{1, n}$ using the kinematic condition (4.11) and the tangential stress condition (4.12) to give

$$
\begin{aligned}
a_{1,0} & =0, \\
a_{1, n} & =\left(i n \omega+2 C k^{2}\right) Z_{1, n}, \quad n \neq 0, \\
c_{1,0} & =0, \\
c_{1, n} & =-2 C k^{2} Z_{1, n}, \quad n \neq 0 .
\end{aligned}
$$

Then the normal stress condition (4.13) is used to eliminate $W_{1, n}$ to give

$$
\begin{array}{rlrl}
k^{2}\left(B k^{2}+1\right) Z_{1,0} & =-\frac{1}{2} a_{0} k^{2} Z_{1, f, 0}, & n & =0, \\
{\left[k\left(2 C k^{2}+i n \omega\right)^{2}-4 C^{2} k^{4} q_{1, n}+k^{2}\left(B k^{2}+1\right)\right] Z_{1, n}} & =-\frac{1}{2} a_{0} k^{2} Z_{1, f, n}, & n \neq 0 .
\end{array}
$$

This is a generalized eigenvalue problem for $a_{0}$ of the form

$$
\mathbf{A Z}=a_{0} \mathbf{B Z},
$$

where $\mathbf{Z}=\left(Z_{1,0}, Z_{1,1}, \ldots, Z_{1, N}\right)^{T}$. This generalized eigenvalue problem is the same as that solved in [15]. The minimum real positive eigenvalue $a_{0}$ gives the critical amplitude of onset of patterns, and the corresponding eigenvector gives the values for $Z_{1, n}$. Hence $W_{1, n}$ can be found from (5.2) and (5.1). These calculations and those that follow were carried out using MATLAB. For the majority of the harmonic calculations, we take $N=20$, and for the subharmonic calculations, we take $N=40$. Doubling the number of modes typically changed the results by less than $0.2 \%$. 
5.2. Linear adjoint problem. The general solution for the linear adjoint problem (4.22) for a fluid of infinite depth is

$$
\begin{aligned}
& W_{0}^{*}(z)=\left(a_{0}^{*}+z c_{0}^{*}\right) e^{k z}, \\
& W_{n}^{*}(z)=a_{n}^{*} e^{k z}+c_{n}^{*} e^{q_{n}^{*} z}, \quad n \neq 0,
\end{aligned}
$$

where

$$
q_{n}^{* 2}=-\frac{i n \omega}{C}+k^{2} .
$$

Note that $q_{n}^{*}$ is the complex conjugate of $q_{1, n}$. This can be solved analytically in terms of $Z_{n}^{*}$ using the adjoint equivalents to the kinematic condition (4.23) and the tangential stress condition (4.24), and after some manipulation we find

$$
\begin{aligned}
& Z_{0}^{*}=-2 C k^{3} W_{0}^{*}(0), \\
& Z_{n}^{*}=\frac{1}{i n d_{n} \omega} W_{n}^{*}(0), \quad n \neq 0,
\end{aligned}
$$

where

$$
d_{n}=\left(k\left(2 C k^{2}-i n \omega\right)^{2}-4 C^{2} k^{4} q_{n}^{*}\right)^{-1} .
$$

Substitution of these expressions for $Z_{n}^{*}$ into the normal stress condition (4.25) gives

$$
\left[k\left(2 C k^{2}-i n \omega\right)^{2}-4 C^{2} k^{4} q_{n}^{*}+k^{2}\left(B k^{2}+1\right)\right] W_{n}^{*}(0)=-\frac{1}{2} a_{0}^{*} k^{2} W_{f, n}^{*}(0), \quad n \neq 0 .
$$

This results in a second generalized eigenvalue problem of the form

$$
\mathbf{A}^{*} \mathbf{W}^{*}=a_{0}^{*} \mathbf{B}^{*} \mathbf{W}^{*} .
$$

As expected, when we solve this, we find $a_{0}=a_{0}^{*}$, and $W_{n}^{*}(0)$ is the complex conjugate of $Z_{1, n}$. Once $W_{n}^{*}$ has been found, then $Z_{n}^{*}$ follows from (5.4).

5.3. Second order problem for stripes and rectangles. Next, we consider the problem for $W_{2, i, n}(z),(4.16)$. The homogeneous equation has the solution

$$
\begin{aligned}
& W_{2, i, 0}(z)=\left(a_{2, i, 0}+z c_{2, i, 0}\right) e^{\tilde{k} z}, \\
& W_{2, i, n}(z)=a_{2, i, n} e^{\tilde{k} z}+c_{2, i, n} e^{q_{2, i, n} z}, \quad n \neq 0,
\end{aligned}
$$

where

$$
\begin{aligned}
\tilde{k}^{2} & =2(1+c) k^{2}, \\
q_{2, i, n}^{2} & =\frac{i n \omega}{C}+\tilde{k}^{2} .
\end{aligned}
$$

In the inhomogeneous equation (4.16), the right-hand side generates terms of the form $\alpha_{l, m} e^{Q_{l, m} z}$. Each of these contributes to the solution a term $\delta_{l, m} e^{Q_{l, m} z}$, where

$$
\delta_{l, m}=\frac{\alpha_{l, m}}{\left(i n \omega-C\left(Q_{l, m}^{2}-\tilde{k}^{2}\right)\right)\left(Q_{l, m}^{2}-\tilde{k}^{2}\right)} .
$$


The general solution to (4.16) is therefore

$$
\begin{aligned}
& W_{2, i, 0}(z)=\left(a_{2, i, 0}+c_{2, i, 0} z\right) e^{\tilde{k} z}+\sum_{l+m=0} \delta_{l, m} e^{Q_{l, m} z}, \\
& W_{2, i, n}(z)=a_{2, i, n} e^{\tilde{k} z}+c_{2, i, n} e^{q_{2, i, n} z}+\sum_{l+m=n} \delta_{l, m} e^{Q_{l, m} z}, \quad n \neq 0 .
\end{aligned}
$$

The coefficients $a_{2, i, n}$ and $c_{2, i, n}$ can be found in terms of $Z_{2, i, n}$, using the tangential stress condition (4.19) and the kinematic condition (4.18), to give

$$
\begin{aligned}
& a_{2, i, n}=\gamma_{1, i, n} Z_{2, i, n}+v_{1, i, n}, \\
& c_{2, i, n}=\gamma_{2, i, n} Z_{2, i, n}+v_{2, i, n},
\end{aligned}
$$

where

$$
\begin{aligned}
\gamma_{1, i, 0} & =0, \\
\gamma_{1, i, n} & =\left(i n \omega+2 C \tilde{k}^{2}\right), \quad n \neq 0, \\
\gamma_{2, i, 0} & =0 \\
\gamma_{2, i, n} & =-2 C \tilde{k}^{2}, \quad n \neq 0,
\end{aligned}
$$

and

$$
\begin{aligned}
v_{1, i, 0} & =S_{2, i, 0}, \\
v_{1, i, n} & =\frac{C}{i n \omega}\left(\left(\frac{i n \omega}{C}+2 \tilde{k}^{2}\right) S_{2, i, n}-S_{3, i, n}\right), \quad n \neq 0, \\
v_{2, i, 0} & =\frac{1}{2 \tilde{k}}\left(S_{3, i, 0}-2 \tilde{k}^{2} S_{2, i, 0}\right), \\
v_{2, i, n} & =\frac{C}{i n \omega}\left(-2 \tilde{k}^{2} S_{2, i, n}+S_{3, i, n}\right), \quad n \neq 0,
\end{aligned}
$$

and

$$
\begin{aligned}
S_{2, i, n}= & -2(1+c) d \sum_{l+m=n} Z_{1, l} D W_{1, m}-\sum_{l+m=n} \delta_{l, m} \\
S_{3, i, n}= & -d \sum_{l+m=n}\left(2(1+c) Z_{1, l} D^{3} W_{1, m}+(3+2 c) \tilde{k}^{2} Z_{1, l} D W_{1, m}\right) \\
& -\sum_{l+m=n} Q_{l, m}^{2} \delta_{l, m}-\tilde{k}^{2} \sum_{l+m=n} \delta_{l, m} .
\end{aligned}
$$

Substitution of the general solution (5.6) into the normal stress condition, (4.20), gives

$$
\begin{aligned}
\tilde{k}^{2}\left(B \tilde{k}^{2}+1\right) Z_{2, i, 0}+\frac{1}{2} a_{0} \tilde{k}^{2} Z_{2, f, i, 0}= & -2 C \tilde{k}^{3} S_{2, i, 0}+S_{1, i, 0} \\
& -3 C \tilde{k}^{2} \sum_{l+m=0} Q_{l, m} \delta_{l, m}+C \sum_{l+m=0} Q_{l, m}^{3} \delta_{l, m},
\end{aligned}
$$




$$
\begin{aligned}
\left(\tilde{k}\left(i n \omega+2 C \tilde{k}^{2}\right) \gamma_{1, i, n}+2 C \tilde{k}^{2} q_{2, i, n} \gamma_{2, i, n}\right. & \\
\left.+\tilde{k}^{2}\left(B \tilde{k}^{2}+1\right)\right) Z_{2, i, n}+\frac{1}{2} a_{0} \tilde{k}^{2} Z_{2, f, i, n}= & -\tilde{k}\left(i n \omega+2 C \tilde{k}^{2}\right) v_{1, i, n}-2 C \tilde{k}^{2} q_{2, i, n} v_{2, i, n} \\
& +S_{1, i, n}-\left(i n \omega+3 C \tilde{k}^{2}\right) \sum_{l+m=n} Q_{l, m} \delta_{l, m} \\
& +C \sum_{l+m=n} Q_{l, m}^{3} \delta_{l, m}, \quad n \neq 0 .
\end{aligned}
$$

This is of the form

$$
\mathbf{A} \mathbf{Z}_{2}=\mathbf{b}
$$

where $\mathbf{Z}_{2}=\left(Z_{2, i, 0}, Z_{2, i, 1}, \ldots, Z_{2, i, N}\right)$. This can be solved for $Z_{2, i, n}$, and hence $W_{2, i, n}$ can be found from (5.7) and (5.6).

5.4. Solvability condition for stripes and rectangles. Once the calculations for the linear, linear adjoint, and second order problem have been completed, the coefficients for the solvability condition are calculated from (4.27), (4.28), (4.29), and (B.1).

5.5. Second order problem and solvability condition for hexagons. The linear and linear adjoint problems for hexagons are the same as for stripes and rectangles. Once $Z_{1}, W_{1}, Z^{*}$, and $W^{*}$ are known, the size of the quadratic coefficient in the amplitude equation for hexagons may be computed from (4.31). The calculations of the second order solution and solvability condition at third order then follow in a very similar fashion to the calculation for rectangles: the same products appear but with different coefficients.

5.6. Evaluating the coefficients of the amplitude equations. In order to compute the stability of the patterns as given in Table 4.1, we need to calculate each of the cubic coefficients $b_{i}$ that appear in the generic amplitude equations (4.1) along with the value of the quadratic coefficient $\tilde{\epsilon}$. From the calculations for rectangles, stripes, and hexagons this may be done as follows. The solvability condition for rectangles and stripes takes the form given in (4.26), that is,

$$
\delta \frac{d A}{d T}=a_{2} \beta A+\gamma A^{3} .
$$

The values of $\delta$ and $\beta$ are independent of the type of pattern considered, but the value of $\gamma$ is not. By comparing the amplitude equations with the equations given for each state given in Table 4.1, we see that when $\gamma$ is computed in the case of stripes, it gives us the value for $b_{1}$. If the solvability condition is calculated for rectangles, then $\gamma$ gives us $b_{1}+b_{4}$, and hence the value of $b_{4}$ (and similarly $b_{5}$ and $b_{6}$ ) may be found. In the case of hexagons, the solvability condition takes the form

$$
\delta \frac{\partial A_{H}}{\partial T}=a_{2} \beta A_{H}+\gamma_{2} A_{H}^{2}+\left(\gamma_{1}+\gamma_{3}\right) A_{H}^{3},
$$

where $\delta, \beta$, and $\gamma_{1}$ are the same as for stripes and $\gamma_{2}$ is given by (4.31) and $\gamma_{3}$ comes from (C.1). By comparing with the amplitude equation for hexagons given in Table 4.1, we see that $\gamma_{3}$ gives the value of $2 b_{2}$. Note that while $b_{1}$ and $b_{2}$ are fixed for a given set of fluid parameters, the values of $b_{4}, b_{5}$, and $b_{6}$ depend on the lattice angle $\theta$.

In the results we present in the next section, we rescale the amplitude equations by letting $A \mapsto \sqrt{\left|\beta / b_{1}\right|} A, A_{H} \mapsto \sqrt{\left|\beta / b_{1}\right|} A_{H}$, and $T \mapsto|\delta / \beta|$ so that the cubic coefficient for stripes $b_{1}$ in the rescaled equations is always \pm 1 . 
(a)

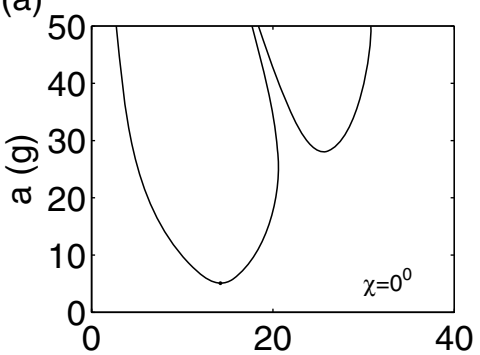

(c)

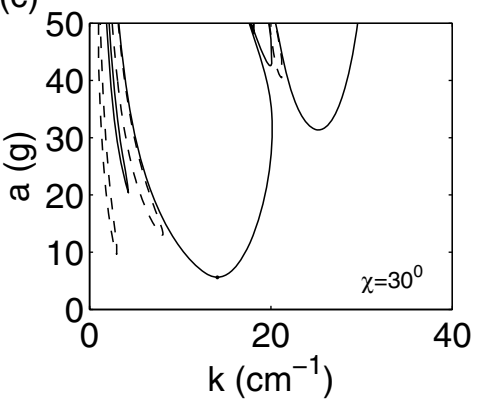

(b)

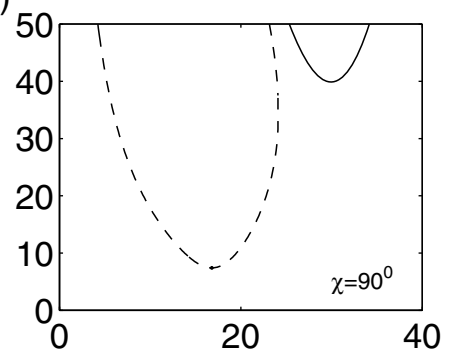

(d)

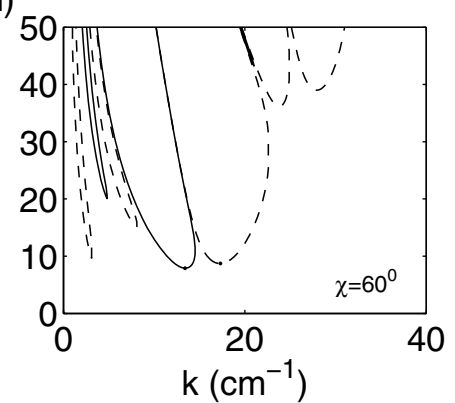

FIG. 6.1. Linear stability curves for different values of $\chi . \rho=0.95 \mathrm{~cm}^{-3}, \nu=20.9 \mathrm{cS}, \sigma=$ 20.6 dyn $/ \mathrm{cm}$. Excitation is a $\left(\cos \chi \cos M_{1} \omega t+\sin \chi \cos M_{2} \omega t\right)$ with $\left(M_{1}, M_{2}\right)=(4,5), \omega=44 \pi$. Harmonic tongues are marked with solid lines and subharmonic tongues with dashed lines.

6. Results. Here we present the results of our calculations for the particular fluid parameters used in the experimental results given in [4].

6.1. Linear stability results. First, the generalized eigenvalue problem (5.3) is solved, and this gives the critical amplitude as a function of $k$. Typical curves are shown in Figure 6.1. These are analogous to those computed by Besson, Edwards, and Tuckerman [15] but focus on the particular parameter values that are used by Kudrolli, Pier, and Gollub [4], namely $\rho=0.95 \mathrm{~cm}^{-3}, \nu=20.9 \mathrm{cS}$, and $\sigma=20.6 \mathrm{dyn} / \mathrm{cm}$. Curves that have a subharmonic response with the excitation are indicated with dotted lines, and those that are harmonic are marked with a solid line. The critical onset occurs at the minimum value of $a$, and this occurs at a critical wavenumber $k_{c}$. For ease of comparison with the experimental results, the curves are plotted in dimensional rather than nondimensional units: with our choice of nondimensionalization, the value of $k_{c}$ is always 1 . The minimum value point is indicated by a dot. The richness of the dynamics that is seen with two-frequency forcing is partly due to the fact that the parameter $\chi$ allows one to tune between the critical value for $a$ occurring for either a harmonic tongue, as is the case for $0^{\circ}<\chi<60^{\circ}$, or a subharmonic tongue, as is the case for $60^{\circ}<\chi<90^{\circ}$. There is a bicritical point that occurs when $\chi$ is approximately $60^{\circ}$. The phase $\phi$ has little impact on the position of the minimum value of the lowest tongue and therefore in the position of the bicritical point (changes in the position of the mimima are typically less than $0.001 \%$ ). The phase does, however, alter the position of the tongues for some of the other harmonics.

Experimentally, it is the onset of patterns as a function of $\chi$ that is observed rather than the linear stability curves directly. In Figure 6.2 , we show how the minimum of the linear stability curves varies with the relative importance of the amplitude of 
(a)

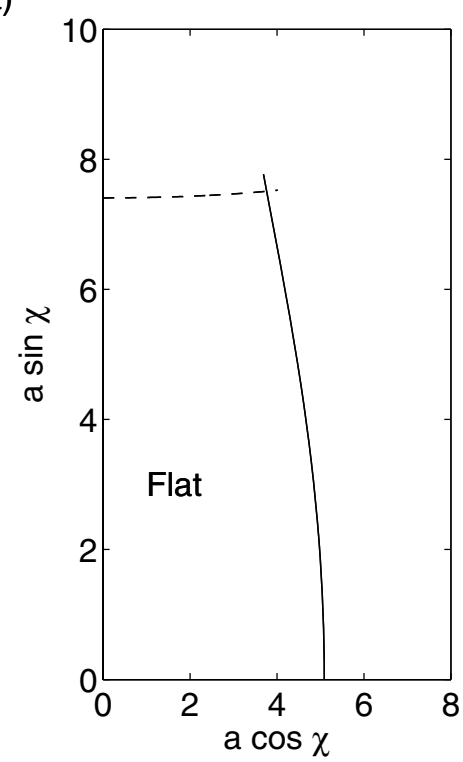

(b)

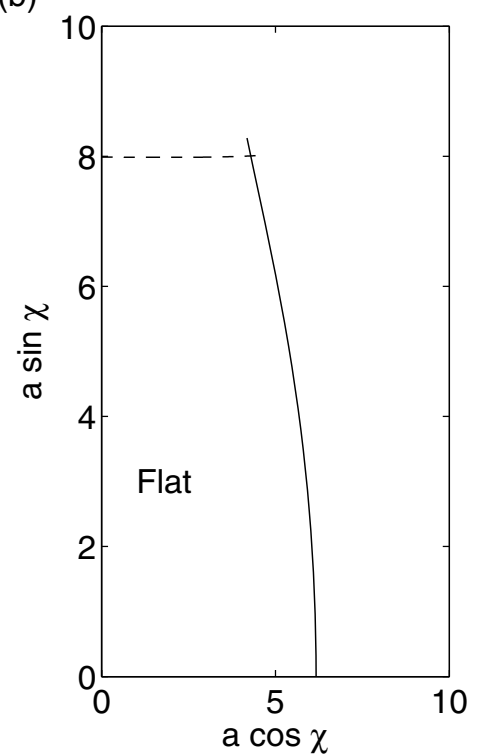

FIG. 6.2. Path of $a_{0}$ as a function of $\chi$. (a) $\left(M_{1}, M_{2}\right)=(4,5), \phi=16^{\circ}$, and $\omega=44 \pi$; (b) $\left(M_{1}, M_{2}\right)=(6,7), \phi=20^{\circ}$, and $\omega=32.88 \pi$.

the two components of the excitation as given by $a \cos \chi$ and $a \sin \chi$. Two cases are shown: Figure 6.2(a) is for the same parameter values as for Figure 6.1. Figure 6.2(b) is for the same fluid parameters but different excitation parameters. These two cases correspond to the two cases considered in detail in Kudrolli, Pier, and Gollub, and the linear stability boundaries compare well with the corresponding experimental results shown in Figures 1 and 6 of their paper [4]. The bicritical point occurs for $\chi=63.4$ in the case $\left(M_{1}, M_{2}\right)=(4,5)$ and $\chi=61.8$ when $\left(M_{1}, M_{2}\right)=(6,7)$. These compare well with the value quoted by Kudrolli, Pier, and Gollub of $\chi=61.5$.

As discussed in the introduction, high viscosity or shallow depth are used to damp modes with a small wavenumber that can make regular patterns hard to observe. The fluid used by Kudrolli, Pier, and Gollub was of moderate viscosity $(C \approx 0.4$ rather than $C \ll 1$ ). How "shallow" a container is depends on the product $k_{c} h$, in particular whether $e^{-k_{c} h}$ is negligible when compared with $e^{k_{c} h}$. For the Kudrolli-Pier-Gollub experiments, $h=0.3 \mathrm{~cm}$, giving $k_{c} \approx 14$ and $e^{-k_{c} h} / e^{k_{c} h} \approx 0.0002$. In the weakly nonlinear calculations, we shall see that it is not just the main harmonic tongue that is of importance but the weakly damped harmonic tongue that has a minimum at $k \approx 6 \mathrm{~cm}^{-1}$. For this tongue, $e^{-k_{c} h} / e^{k_{c} h} \approx 0.03$. Since this is also small, we believe that an infinite depth approximation is reasonable. Consequently, in the nonlinear results that we present below, the calculations are performed only for infinite depth.

6.2. Single-frequency results $\left(\chi=0^{\circ}\right.$ and $\left.\chi=90^{\circ}\right)$. The two-frequency excitation term we are interested in is of the form

$$
f(t)=f_{2}(t)=\cos (\chi) \cos \left(M_{1} \omega t\right)+\sin (\chi) \cos \left(M_{2} \omega t+\phi\right) .
$$

When $\chi=0^{\circ}$ or $90^{\circ}$, this reduces to a single-frequency excitation. When $\chi=0^{\circ}$, this corresponds to a pure $M_{1}$ excitation, and when $\chi=90^{\circ}$, this is a pure $M_{2}$ excitation. 


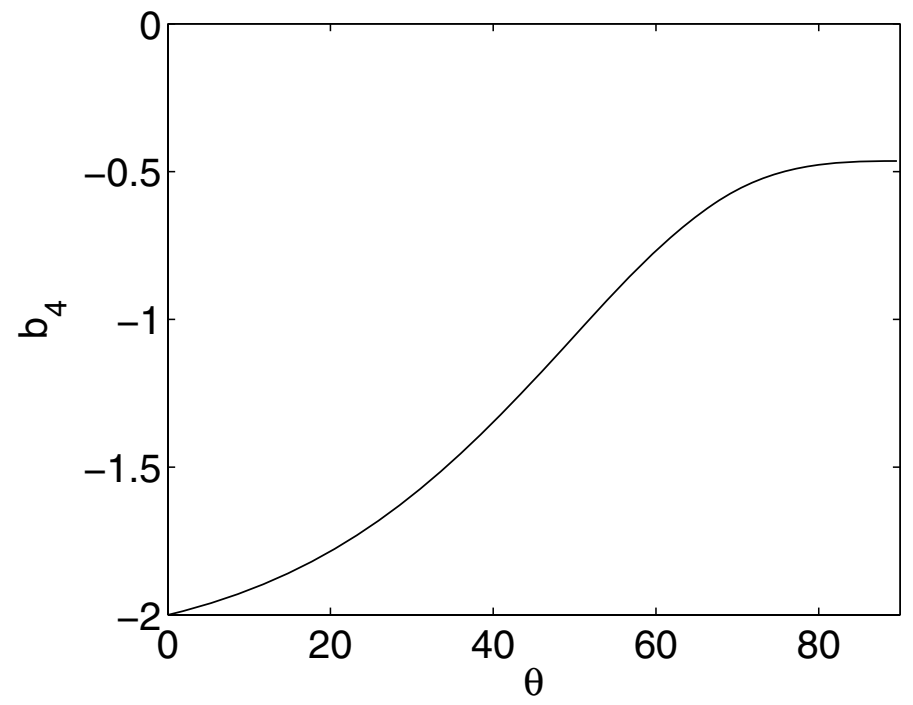

FIG. 6.3. For $\left(M_{1}, M_{2}\right)=(4,5)$ and $\chi=0^{\circ}$ the value of the coefficient $b_{4}$ as a function of the lattice angle $\theta$.

Since the two cases we consider have $M_{1}$ even and $M_{2}$ odd, the pure $M_{1}$ response is harmonic and the pure $M_{2}$ response is subharmonic. In each case, the overall picture is similar. The quadratic term in the amplitude equations is zero and we find the following:

- All the eigenvalues for hexagons given in Table 4.1 are negative for all values of the lattice angle $\theta$. This suggests that hexagons are a stable state.

- Stripes and superlattice patterns have at least one unstable eigenvalue.

- Rectangular patterns are stable, on the lattice on which they occur, if they are "sufficiently square."

This last point may be seen by considering the eigenvalues of the family of rectangles $R_{h 1, m, n}$ listed in Table 4.1. The eigenvalues $b_{1}+b_{4}, \mu_{1}$, and $\mu_{2}$ are all negative. The remaining eigenvalue, $b_{1}-b_{4}$, is negative only if the lattice angle $\theta$ is sufficiently large. In the case $\left(M_{1}, M_{2}\right)=(4,5)$ and $\chi=0^{\circ}$ in Figure 6.3, we plot the scaled value of $b_{4}$ as a function of the lattice angle $\theta$. Since in the scaled units $b_{1}=-1, b_{1}-b_{4}<0$ only if $\theta>52^{\circ}$. The aspect ratio of the rectangles is given by $\sqrt{(1-\cos \theta) /(1+\cos \theta)}$ so that stability for rectangles with $\theta>52^{\circ}$ means that rectangles with an aspect ratio between 0.48 and 1 are stable (on the lattice on which they occur).

By considering the eigenvalues on spatially periodic lattices alone, we find that both hexagons and rectangles are possible stable states. If we find the values of the Lyapunov function $\mathcal{F}$ (see Table 4.2) for hexagons and rectangles, then we find that those rectangles with an aspect ratio closest to 1 have the lowest value.

The results are similar for the three other single frequency cases that are relevant to the bifurcation sets shown in Figure 6.2, namely $\left(M_{1}, M_{2}\right)=(4,5)$ and $\chi=90^{\circ}$ and $\left(M_{1}, M_{2}\right)=(6,7)$ and $\chi=0^{\circ}$ or $\chi=90^{\circ}$. The conclusion is that the calculations for solutions on periodic lattices show that stripe patterns are unstable. Hexagons and "sufficiently square" rectangular patterns are relatively stable states with square patterns having the lowest value of the Lyapunov function. This suggests that square patterns will be observed at the points where $\chi=0^{\circ}$ and $\chi=90^{\circ}$. These square 

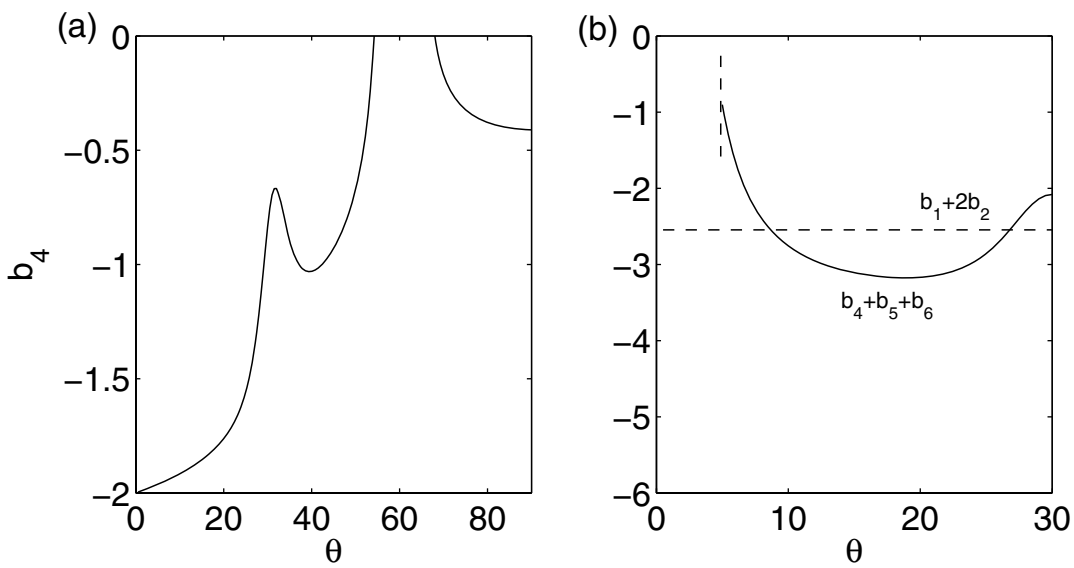

FIG. 6.4. $\left(M_{1}, M_{2}\right)=(4,5)$ and $\chi=60^{\circ}, \phi=20^{\circ}$ : (a) The value of the coefficient $b_{4}$ as a function of the lattice angle $\theta$. At $\theta=60^{\circ}$ quadratic resonance occurs, and the calculation for $b_{4}$ is not valid. For this reason, this point has been excluded from the calculation. (b) The value of the coefficient $b_{4}+b_{5}+b_{6}$ compared with $b_{1}+2 b_{2}$ as a function of the lattice angle $\theta$. Just as $b_{4}$ has $a$ singularity at $60^{\circ}, b_{5}$ has a singularity at $0^{\circ}$, and so the region around $0^{\circ}$ has been excluded.

patterns will be harmonic when $\chi=0^{\circ}$ and subharmonic when $\chi=90^{\circ}$. This agrees with the experimental findings of Kudrolli, Pier, and Gollub.

Note that if we use the same parameter values as used by Chen and Viñals in [22] in their single-frequency study, then we get excellent agreement with their work.

6.3. Two-frequency results $\left(0<\chi<\mathbf{9 0}^{\circ}\right)$. For two-frequency excitation, we have performed a systematic study of the coefficients $\tilde{\epsilon}, b_{1}, b_{2}, b_{3}, b_{4}, b_{5}$, and $b_{6}$ as a function of $\chi$ and the lattice angle $\theta$ for the same fluid parameters as used above and for the two cases $\left(M_{1}, M_{2}\right)=(4,5)$ and $\left(M_{1}, M_{2}\right)=(6,7)$. In the first case, most results are presented for $\phi=16^{\circ}$ and in the second case for $\phi=20^{\circ}$ : these are the values for which the majority of the results in [4] are presented. We focus on the onset of harmonic patterns since along the subharmonic branch squares the absence of the quadratic term means that hexagons and rectangles remain the only stable states, with squares having the lowest energy.

From the values of the coefficients we have computed the stability of the different planforms based on the eigenvalues for each state given in Table 4.1 as a function of the lattice angle. If the eigenvalues indicate that the state is relatively stable, then we compute the value of the Lyapunov function, as given in Table 4.2.

When $\chi$ is zero, $b_{4}$ increases monotonically with $\theta$, as shown in Figure 6.3. However, this changes as $\chi$ is increased and peaks develop. In Figure 6.4(a), we plot $b_{4}(\theta)$ for $\left(M_{1}, M_{2}\right)=(4,5)$ and for $\chi=60^{\circ}, \phi=16^{\circ}$. The angle $\theta=60^{\circ}$ has been excluded because this corresponds to the quadratic resonance point, and the calculation for rectangles breaks down here. From Table 4.1 it can be seen that for superlattice patterns to become stable, one needs $b_{4}+b_{5}+b_{6}-b_{1}-2 b_{2}>0$. This same quantity causes the destabilization of hexagons. In Figure $6.4(\mathrm{~b})$, we plot $b_{4}+b_{5}+b_{6}$ along with $b_{1}+2 b_{2}$. For most values of $\theta$, and so on most lattices, $b_{4}+b_{5}+b_{6}-b_{1}-2 b_{2}<0$, and superlattice patterns are unstable. However, the peak in $b_{4}(\theta)$ at $31.8^{\circ}$ leads to a small region centered at $30^{\circ}$ for which $b_{4}+b_{5}+b_{6}-b_{1}-2 b_{2}>0$, and it is possible for superlattice patterns to be stable.

The corresponding graphs of $b_{4}$ and $b_{4}+b_{5}+b_{6}$ for $\left(M_{1}, M_{2}\right)=(6,7)$ are 

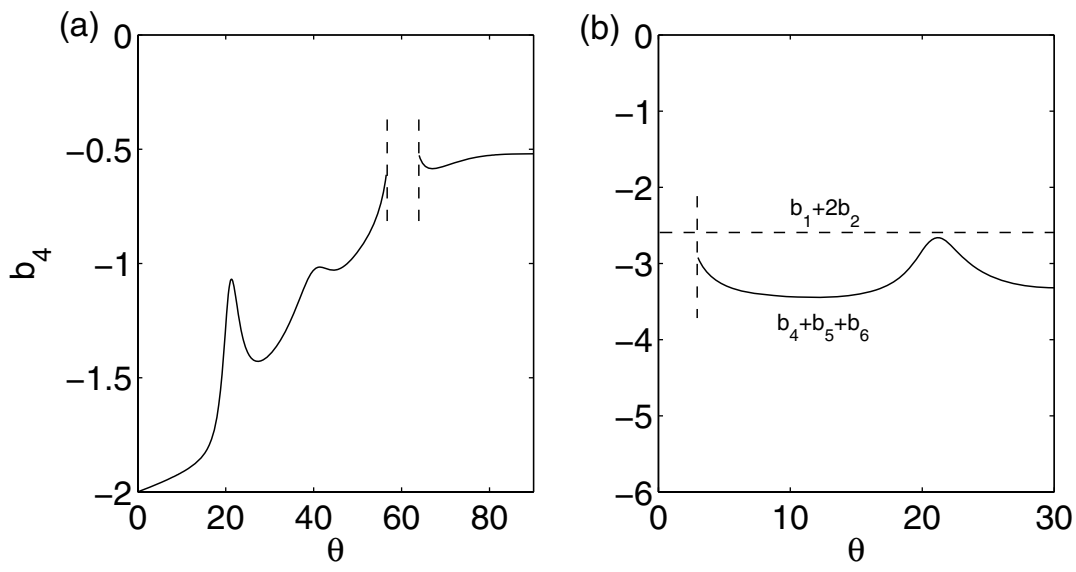

FIG. 6.5. $\left(M_{1}, M_{2}\right)=(6,7)$ and $\chi=60^{\circ}$ : (a) The value of the coefficient $b_{4}$ as a function of the lattice angle $\theta$; (b) the value of the coefficient $b_{4}+b_{5}+b_{6}$ compared with $b_{1}+2 b_{2}$ as a function of the lattice angle $\theta$.

shown in Figure 6.5. In this case, there is a peak in $b_{4}$ at $21.2^{\circ}$, and this leads to a peak in $b_{4}+b_{5}+b_{6}$ with a maximum at $21.2^{\circ}$. Note that even at $\chi=60^{\circ}$, $b_{4}+b_{5}+b_{6}-b_{1}-2 b_{2}<0$, and all superlattice patterns are unstable. It is only once, $\chi>61^{\circ}$, that stable superlattice patterns occur, the first to stabilize being those that occur on a lattice with lattice angle $\theta=21.2^{\circ}$.

We have shown for $\chi=0^{\circ}$ that both hexagons and rectangles may be stable with rectangles having the lowest value of the Lyapunov function. Figure 6.4 suggests that in the case $\left(M_{1}, M_{2}\right)=(4,5)$, stable superlattice patterns may occur for $\chi=60^{\circ}$. In Figure 6.6(a), we show a bifurcation set that summarizes the regions where different states are stable for the $\lambda, \chi$ plane. Note that hexagons are a planform that exists on all the hexagonal lattices and so that where hexagons are shown as stable they are stable to perturbations on all hexagonal lattices. Where they are unstable, there is at least one lattice on which they are unstable. The peak in $b_{4}(\theta)$ at $30^{\circ}$ means that the maximal region of instability for hexagons occurs as $\theta_{h} \rightarrow 30^{\circ}$. For rectangles and superlattices, the story is more complicated. Different lattices support different superlattice patterns and different rectangles. Since it is on lattices with $\theta \rightarrow 30^{\circ}$ that hexagons become unstable first (and superlattices onset first), we plot the regions for the stability of rectangles and superlattice patterns for the specific case $\theta=30^{\circ}$.

At the actual value of $\theta=30^{\circ}$ the center manifold reduction that leads to the amplitude equations is not formally valid, as discussed in [24], and the "superlattice patterns" are in fact quasipatterns. However, in practical terms, there is little real difference between taking a periodic lattice that has a lattice angle close to $30^{\circ}$ and taking $30^{\circ}$ itself: although on the periodic lattice the amplitude equations may be formally justified, the spectral gap between critical and noncritical eigenvalues will be small, and thus the formal region of validity for the center manifold is likely to be small. Visually, it is impossible to distinguish between a quasipattern and a superlattice pattern with a very large lengthscale. Similarly, the regions of stability would be indistinguishable whether we took $\theta=30^{\circ}$ or a value of $\theta$ close to $30^{\circ}$ that results in a spatially periodic lattice.

As shown in Figure 6.6(a), for most values of $\lambda$ and $\chi$, there is bistability where more than one pattern is stable. In Figure 6.6(b), we show which of the stable 
(a)

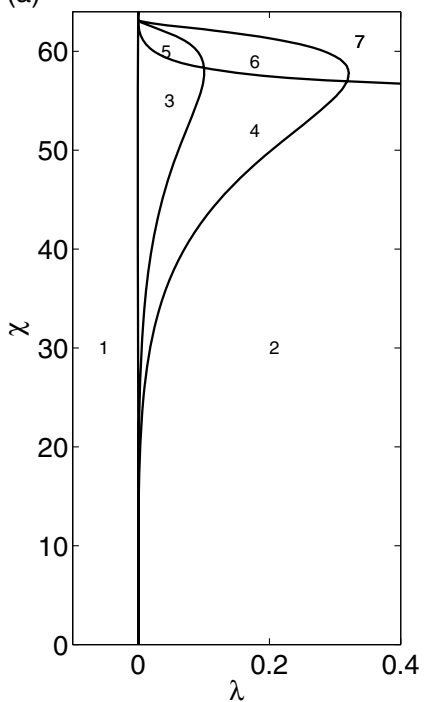

(b)

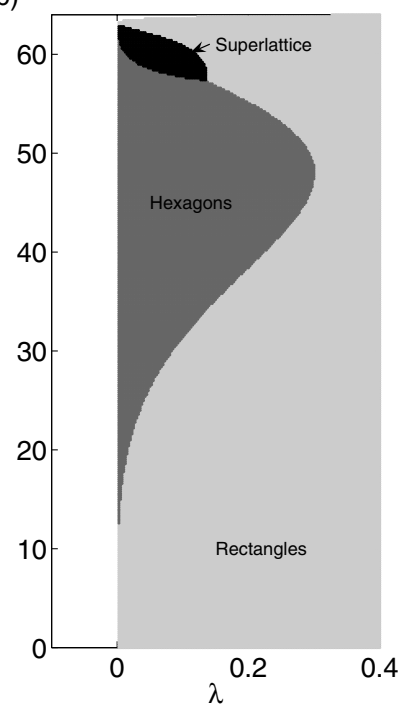

FIG. 6.6. Results in the $\lambda, \chi$ plane for $\left(M_{1}, M_{2}\right)=(4,5)$ and $\phi=16^{\circ}$. (a) Eigenvalue results. The regions correspond to 1 . trivial state stable, 2. stable rectangles and hexagons, 3. stable hexagons and superlattice patterns, 4. stable rectangles, hexagons, and superlattice patterns, 5. stable superlattice patterns, 6. stable superlattice patterns and rectangles, 7. stable rectangles. (b) Patterns with the lowest energy as computed from the Lyapunov function. Black: superlattice patterns. Dark grey: hexagons. Light grey: rectangles.

patterns has the lowest value of the Lyapunov function. What we find is that, for small values of $\chi$, rectangles and hexagons are both stable, with rectangles being the most stable state. Note that it is the $R_{h 3, m, n}$ rectangles that are stable, and as $\theta \rightarrow 30^{\circ}$, the aspect ratio of these particular rectangles tends to 1 . As $\chi$ increases, the value of $\tilde{\epsilon}$ increases from zero, and this results in the destabilization of the rectangles so that hexagons become the preferred state at onset. Near the bicritical point, hexagons are themselves destabilized to superlattice patterns, and there are regions where superlattice patterns are the only stable state. A typical bifurcation diagram for $\left(M_{1}, M_{2}\right)=(4,5)$ at $\chi=60^{\circ}$ is shown in Figure 6.7. Note that the values of $\tilde{\epsilon}$ are small so that higher order correction terms for the position of the secondary bifurcation points are unlikely to affect the overall qualitative bifurcation sequence.

The analogous bifurcation set and energy diagram for the case $\left(M_{1}, M_{2}\right)=(6,7)$ are shown in Figure 6.8. In this case, the lattice angle $\theta=21.2^{\circ}$ has been used since in the case $(6,7)$, it is on this lattice that hexagons are destabilized first. On this lattice, the stable rectangles have aspect ratio 0.86 . The overall picture is similar to that for $(4,5)$ but with the transitions from rectangle to hexagon and hexagon to superlattice pattern occurring for larger values of $\chi$.

Figures 6.6 and 6.8 compare well with the experimental results of Kudrolli, Pier, and Gollub. As is suggested by our results, they find that rectangles are stable for low values of $\chi$ giving way to hexagons as $\chi$ is increased. As for our theoretical results, the transition from rectangles to hexagons occurs for higher values of $\chi$ in the $(6,7)$ case than in the $(4,5)$ case. In both cases, there are superlattice/quasipatterns near the bicritical point.

Kudrolli, Pier, and Gollub also investigated the dependence of their results on the 


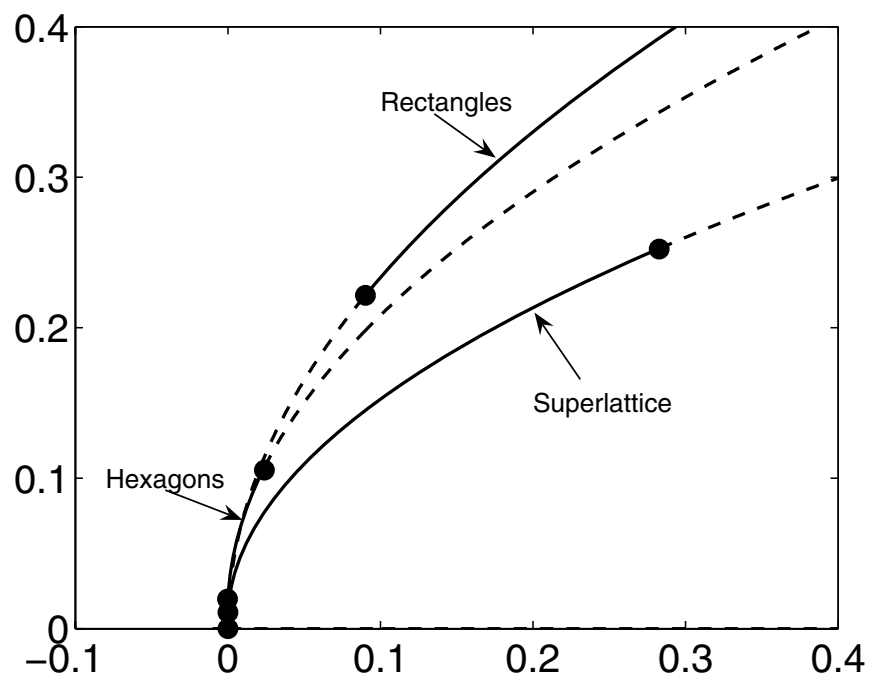

FIG. 6.7. Bifurcation diagram for $\left(M_{1}, M_{2}\right)=(4,5)$ and $\chi=60^{\circ}, \phi=16^{\circ}$. Stable branches are shown by a solid line and unstable lines by a dotted line. Only branches where some part is stable are shown.
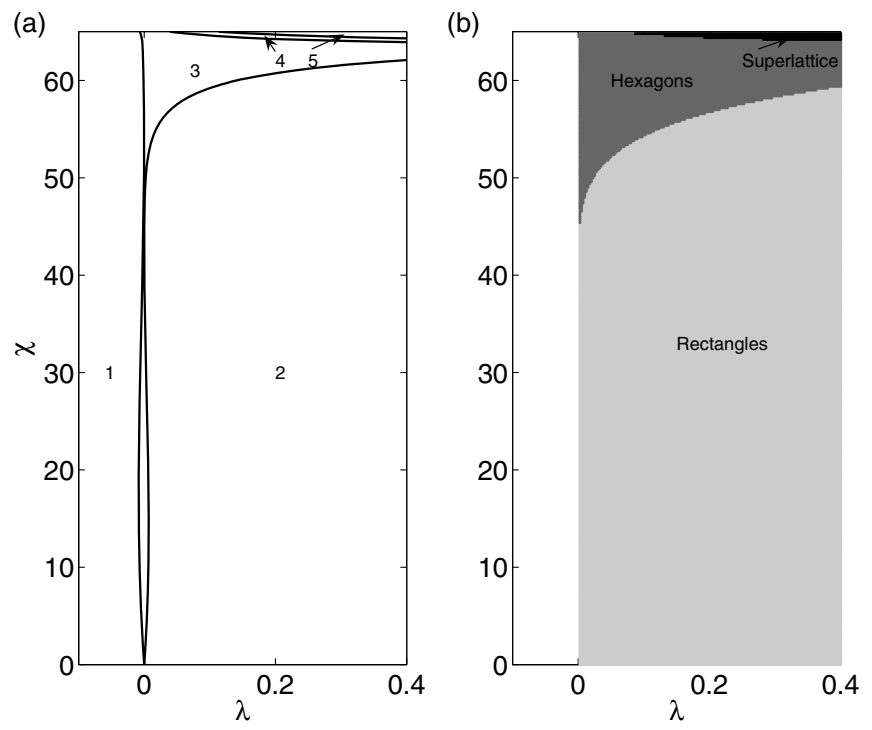

FIG. 6.8. Results in the $\lambda, \chi$ plane for $\left(M_{1}, M_{2}\right)=(6,7)$. (a) The regions correspond to 1 . trivial state stable, 2. stable rectangles and hexagons, 3. hexagons, 4. stable hexagons and superlattice patterns, 5. stable superlattice patterns. (b) Patterns with the lowest energy as computed from the Lyapunov function. Black: superlattice patterns; dark grey: hexagons; light grey: rectangles.

phase $\phi$ for $\chi=61^{\circ}$. In the $(6,7)$ case, they found relatively little phase dependence. In the $(4,5)$ case, they found that the largest region of superlattice patterns was for angles of $\phi$ close to $16^{\circ}$, but they also found that for some values of $\phi$ there were 

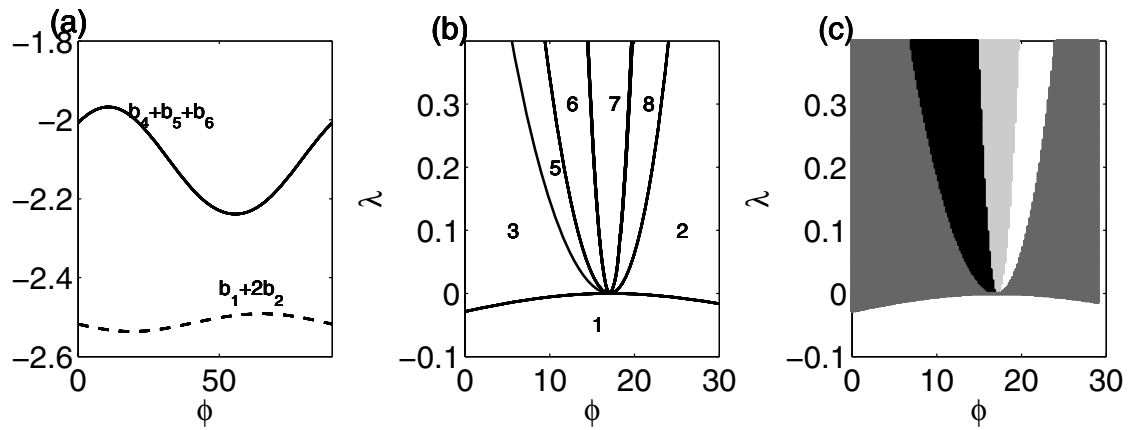

Fig. 6.9. $\left(M_{1}, M_{2}\right)=(4,5), \chi=61^{\circ}, \theta=30^{\circ}$ : (a) Dependence of $b_{1}+2 b_{2}$ and $b_{4}+b_{5}+b_{6}$ on $\phi$. (b) Bifurcation set: 1. trivial state stable, 2. stable rectangles and hexagons, 3. stable hexagons and superlattice patterns, 5. stable superlattice patterns, 6. stable superlattice patterns and rectangles, 7. stable rectangles, 8. no state stable. (c) Planforms with lowest energy as a function of $\phi$ and $\lambda$. Black: superlattice; dark grey: hexagons; light grey: rectangles; white (for $\lambda>0$ ): no stable state found.

no stable states near onset and for others the hexagons bifurcated to a pattern they called superlattice II. We cannot hope to capture this latter transition in our study since these patterns are time periodic and our amplitude equations have a gradient structure. A theoretical explanation for these patterns was given in [31]. Nevertheless, we illustrate how the phase does effect our results in Figure 6.9 for $(4,5)$ and $\chi=61^{\circ}$. First, in Figure 6.9(a), the quantities $b_{1}+2 b_{2}$ and $b_{4}+b_{5}+b_{6}$ are shown. These are $\pi / 2$ periodic functions of $\phi$. The consequence is that the regions of stability of different patterns depend on $\phi$, as shown by the bifurcation set in Figure 6.9(b) and the corresponding plot of the Lyapunov function in Figure 6.9(c) for the angle $\theta=30^{\circ}$. These show that it is for phases close to $16^{\circ}$ that stable superlattice patterns occur closest to onset. We also find values of the phase for which there are no stable spatially periodic pattern near onset (the white wedge region in Figure 6.9).

Overall, the results of Figures 6.4 through 6.8 show that the cases $\left(M_{1}, M_{2}\right)=$ $(4,5)$ and $\left(M_{1}, M_{2}\right)=(6,7)$ are broadly similar. The experimental results show similar bifurcation sequences in both cases but very different forms for the planforms: in the case $(6,7)$, superlattice patterns are observed with an easily visible regular periodic structure with two wavelengths. In [4], these are referred to as superlattice-I patterns. In the $(4,5)$ case, quasipatterns are observed. The key difference in the two cases is the lattice angle for which stable superlattice patterns are possible. In [18], it was argued that the stabilization of the superlattice-I patterns followed from a resonant triad formed by the harmonic tongue with one of the weakly damped harmonic tongues. This was supported by a peak in the value of the rhombic coefficient $\left(b_{4}\right.$ here) at around $22^{\circ}$ for the Zhang-Vinãls model. In Figures 6.5 and 6.8, we have seen that the same mechanism operates in the full Navier-Stokes equations. Furthermore, it is the same fundamental mechanism that is at play in the $(4,5)$ case, as shown in Figures 6.4 and 6.6 , where now it is a peak in $b_{4}$ at $32.8^{\circ}$ which is significant. This peak can again be traced to a resonant triad between the main harmonic mode and the first (that is, smallest $k$ ) weakly damped harmonic mode. The consequence is that while the analysis for the $(6,7)$ case suggests that a superlattice pattern with angle close to $21.2^{\circ}$, such as that shown in Figure 6.10(a), will occur, for the $(4,5)$ case a superlattice pattern/quasipattern with angle close to $30^{\circ}$, such as that shown in Figure 6.10(b), will occur. 
(a)

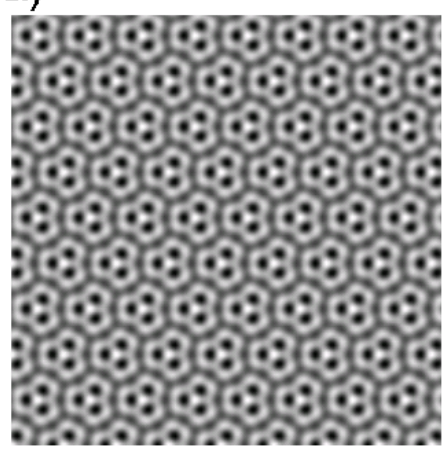

(b)

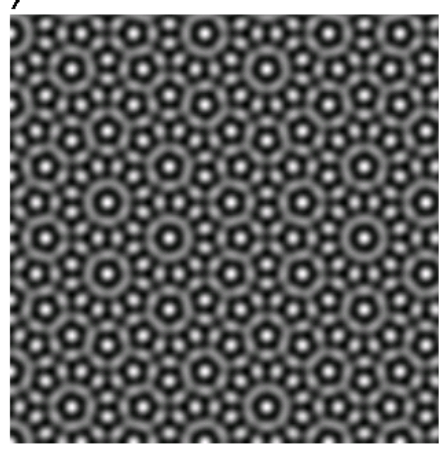

FIG. 6.10. Typical superlattice patterns. Superlattice patterns have the form $\mathbf{z}=$ $\left(A_{S H}, A_{S H}, A_{S H}, A_{S H}, A_{S H}, A_{S H}\right)$; the associated planform has the form $A_{S H}\left(\cos \left(\mathbf{K}_{\mathbf{1}_{\mathbf{h}}} \cdot \mathbf{r}+\psi\right)+\right.$ $\left.\cos \left(\mathbf{K}_{\mathbf{2}_{\mathbf{h}}} \cdot \mathbf{r}+\psi\right)+\cos \left(\mathbf{K}_{\mathbf{3}_{\mathbf{h}}} \cdot \mathbf{r}+\psi\right)+\cos \left(\mathbf{K}_{\mathbf{4}_{\mathbf{h}}} \cdot \mathbf{r}+\psi\right)+\cos \left(\mathbf{K}_{\mathbf{5}_{\mathbf{h}}} \cdot \mathbf{r}+\psi\right)+\cos \left(\mathbf{K}_{\mathbf{6}_{\mathbf{h}}} \cdot \mathbf{r}+\psi\right)\right)$, where $\mathbf{r}=(x, y)$. (a) For $\theta \approx 21.2^{\circ}, \psi=2 \pi / 3$. (b) For $\theta \approx 30^{\circ}, \psi=0$. The superlattice pattern for $\psi=0$ has a hexagonal rather than a triangular symmetry. Of these two states, only one is stable, but which one is not determined at cubic order. The experimental patterns for the $(6,7)$ case clearly show a triangular structure, and it was this that motivated the theoretical work of [27] on the $\psi=2 \pi / 3$ superlattice pattern.

Note that there is a peak in the value of the coefficient $b_{4}$ for values of $\chi$ far from the bicritical point. For example, there is still a distinct peak in $b_{4}$ at approximately $21^{\circ}$ for $\chi=30^{\circ}$ in the $(4,5)$ case. This can be related to the fact that the weakly damped harmonic tongue involved in the triad resonance is still prominent, as seen in Figure 6.1(c). However, it is only near the bicritical point that $b_{4}+b_{5}+b_{6}-b_{1}-2 b_{2}$ becomes positive, allowing superlattice patterns to stabilize.

7. Conclusion. In this paper, we have derived the form of the weakly nonlinear problem for a finite depth of fluid that is subject to a vertical oscillation from the full Navier-Stokes equations. Using the ideas of symmetry for patterns that tessellate the plane, we have found the coefficients of the amplitude equations and calculated the consequences for stability of different spatially periodic patterns in the infinite depth case. We have focused on the particular parameters that were used in experimental results presented in [4]. Good agreement has been found between the predictions of the weakly nonlinear analysis and the experimental results, without the use of any fitted parameters.

In the future, there are many interesting questions relating to regular patterns in the Faraday problem that we plan to use our method to explore. Our current code will allow us to perform a careful comparison between the Zhang-Vinals equations and the full Navier-Stokes equations for varying viscosity. We will also be able to investigate how the coefficients of the amplitude equations scale with the different parameters and compare with the scaling arguments given in [20] for multiple frequency forcing. This will enable us to see the degree to which the arguments for the control of patterns through multiple frequency in the weak viscosity case carry over to moderate and large viscosity. (With our formulation, it is straightforward to include more than two frequency components.)

The solvability condition is valid for both finite and infinite depth, although our subsequent calculations were carried out only for infinite depth. Our future plans also include coding up the finite depth case. This will allow us to explore how the coef- 
ficients vary with depth and enable comparison with experimental results in shallow containers to be made.

Meanwhile, the Faraday wave experiment continues to be a rich source for striking and intriguing patterns, as shown by the recent results of Epstein and Fineberg [32].

Appendix A. Form for the horizontal components of the velocity. The form of the horizontal velocity components can be derived from the form of the vertical velocity and equations (2.1). Hence, the horizontal velocity components for rectangles at first order are

$$
\begin{aligned}
& u_{1}=\frac{i A_{R}}{k}\left[e^{i k x}+c e^{i k(c x+s y)}-c . c .\right] \sum_{n} D W_{1, n}(z) e^{i(n \omega+\alpha) \tau}, \\
& v_{1}=\frac{i s A_{R}}{k}\left[e^{i k(c x+s y)}-c . c .\right] \sum_{n} D W_{1, n}(z) e^{i(n \omega+\alpha) \tau} .
\end{aligned}
$$

Horizontal velocity components for rectangles at second order are

$$
\begin{aligned}
u_{2}= & \frac{i}{2 k} A_{R}^{2}\left[e^{2 i k x}+c e^{2 i k(c x+s y)}-c . c .\right] \sum_{n} D W_{2,1, n}(z) e^{i(n \omega+2 \alpha) \tau} \\
& +\frac{i}{2 k} A_{R}^{2}\left[e^{i k[(1+c) x+s y]}-c . c .\right] \sum_{n} D W_{2,2, n}(z) e^{i(n \omega+2 \alpha) \tau} \\
& +\frac{i}{2 k} A_{R}^{2}\left[e^{i k[(1-c) x-s y]}-c . c .\right] \sum_{n} D W_{2,3, n}(z) e^{i(n \omega+2 \alpha) \tau}, \\
v_{2}= & \frac{i s}{2 k} A_{R}^{2}\left[e^{2 i k(c x+s y)}-c . c .\right] \sum_{n} D W_{2,1, n}(z) e^{i(n \omega+2 \alpha) \tau} \\
& +\frac{i s}{2 k(1+c)} A_{R}^{2}\left[e^{i k[(1+c) x+s y]}-c . c .\right] \sum_{n} D W_{2,2, n}(z) e^{i(n \omega+2 \alpha) \tau} \\
& -\frac{i s}{2 k(1-c)} A_{R}^{2}\left[e^{i k[(1-c) x-s y]}-c . c .\right] \sum_{n} D W_{2,3, n}(z) e^{i(n \omega+2 \alpha) \tau} .
\end{aligned}
$$

Horizontal velocity components for hexagons at first order are

$$
\begin{aligned}
& u_{1}=\frac{i A_{H}}{k}\left[e^{i k x}-\frac{1}{2} e^{i k(-x+\sqrt{3} y) / 2}-\frac{1}{2} e^{i k(-x-\sqrt{3} y) / 2}-c . c .\right] \sum_{n} D W_{1, n}(z) e^{i(n \omega+\alpha) \tau}, \\
& v_{1}=\frac{i \sqrt{3} A_{H}}{2 k}\left[e^{i k(-x+\sqrt{3} y) / 2}-e^{i k(-x-\sqrt{3} y) / 2}-c . c .\right] \sum_{n} D W_{1, n}(z) e^{i(n \omega+\alpha) \tau} .
\end{aligned}
$$

Horizontal velocity components for hexagons at second order are

$$
\begin{aligned}
u_{2}= & \frac{i A_{H}^{2}}{2 k}\left[e^{2 i k x}-\frac{1}{2} e^{i k(-x+\sqrt{3} y)}-\frac{1}{2} e^{i k(-x-\sqrt{3} y)}-c . c .\right] \sum_{n} D W_{2,1, n}(z) e^{i(n \omega+2 \alpha) \tau} \\
& +\frac{i A_{H}^{2}}{2 k}\left[e^{i k(3 x+\sqrt{3} y) / 2}+e^{i k(3 x-\sqrt{3} y) / 2}-c . c .\right] \sum_{n} D W_{2,2, n}(z) e^{i(n \omega+2 \alpha) \tau}, \\
v_{2}= & \frac{i \sqrt{3} A_{H}^{2}}{4 k}\left[e^{i k(-x+\sqrt{3} y)}-e^{i k(-x-\sqrt{3} y)}-c . c .\right] \sum_{n} D W_{2,1, n}(z) e^{i(n \omega+2 \alpha) \tau} \\
& +\frac{i A_{H}^{2}}{\sqrt{3} k}\left[e^{i k \sqrt{3} y}+\frac{1}{2} e^{i k(3 x+\sqrt{3} y) / 2}-\frac{1}{2} e^{i k(3 x-\sqrt{3} y) / 2}-c . c .\right] \sum_{n} D W_{2,2, n}(z) e^{i(n \omega+2 \alpha) \tau} .
\end{aligned}
$$


Appendix B. Cubic coefficient for rectangles and stripes.

$$
\begin{aligned}
& \gamma_{i}=-\sum_{l, m, n}^{(2)} Z_{l}^{*}\left[Z_{1, m} D W_{2, i, n}(0)-2 c D W_{1, m}(0) Z_{2, i, n}\right] \\
& -\sum_{l, m, n}^{(2)} W_{l}^{*}(0)\left[W_{1, m}(0) D^{2} W_{2, i, n}(0)-2 c D^{2} W_{1, m}(0) W_{2, i, n}(0)\right] \\
& +2 k^{2} \sum_{l, m, n}^{(2)} W_{l}^{*}(0)\left[Z_{1, m} D P_{2, i, n}(0)+\frac{1}{4 d} Z_{1, m} D P_{2,4, n}(0)+D P_{1, m}(0) Z_{2, i, n}\right] \\
& -2 C k^{2} \sum_{l, m, n}^{(2)} W_{l}^{*}(0)\left[Z_{1, m} D^{2} W_{2, i, n}(0)+2 D^{2} W_{1, m}(0) Z_{2, i, n}\right] \\
& -\sum_{l, m, n}^{(2)} W_{l}^{*}(0)\left[D W_{1, m}(0) D W_{2, i, n}(0)-4(1+c) C k^{4} Z_{1, m} W_{2, i, n}(0)\right] \\
& -C \sum_{l, m, n}^{(2)} D W_{l}^{*}(0)\left[Z_{1, m} D^{3} W_{2, i, n}(0)-2 c D^{3} W_{1, m}(0) Z_{2, i, n}\right] \\
& -2 C k^{2} \sum_{l, m, n}^{(2)} D W_{l}^{*}(0)\left[\left(3+3 c+c^{2}-s^{2}\right) D W_{1, m}(0) Z_{2, i, n}-2 c Z_{1, m} D W_{2, i, n}(0)\right] \\
& -\sum_{l, m, n, j}^{(3)} Z_{l}^{*} Z_{1, m} Z_{1, n} D^{2} W_{1, j}(0) \\
& +\sum_{l, m, n, j}^{(3)} W_{l}^{*}(0) Z_{1, m} Z_{1, n}\left[B k^{6}\left(3-2 s^{2}\right) Z_{1, j}+4 C k^{4}\left(2+c^{2}\right) D W_{1, j}(0)\right. \\
& \left.-2 C k^{2} D^{3} W_{1, j}(0)+3 k^{2} D^{2} P_{1, j}(0)\right] \\
& -C \sum_{l, m, n, j}^{(3)} D W_{l}^{*}(0) Z_{1, m} Z_{1, n}\left[\left(9-4 s^{2}\right) k^{2} D^{2} W_{1, j}(0)+D^{4} W_{1, j}(0)\right] \\
& -\sum_{l, m, n}^{(2)} \int_{-h / l}^{0} W_{l}^{*}(z)\left[2 k^{2}(2+c) D W_{1, m}(z) W_{2, i, n}(z)+3 k^{2} W_{1, m}(z) D W_{2, i, n}(z)\right] d z \\
& -\sum_{l, m, n}^{(2)} \int_{-h / l}^{0} W_{l}^{*}(z)\left[(2 c-1) D^{2} W_{1, m}(z) D W_{2, i, n}(z)-2 D W_{1, m}(z) D^{2} W_{2, i, n}(z)\right] d z \\
& \left.+\sum_{l, m, n}^{(2)} \int_{-h / l}^{0} W_{l}^{*}(z)\left[W_{1, m}(z) D^{3} W_{2, i, n}(z)-2 c D^{3} W_{1, m}(z) W_{2, i, n}(z)\right)\right] d z .
\end{aligned}
$$

The following notation has been used for the sums in (4.27), (4.28), (4.29), and (B.1):

$$
\begin{aligned}
\sum_{l, m}^{(1)} & = \begin{cases}l+m=0 \quad(\alpha=0), \\
l+m+1=0 & (\alpha=\omega / 2),\end{cases} \\
\sum_{l, m, n}^{(2)} & = \begin{cases}l+m+n=0 & (\alpha=0), \\
l+m+n+2=0 & (\alpha=\omega / 2),\end{cases} \\
\sum_{l, m, n, j}^{(3)} & = \begin{cases}l+m+n+j=0 & (\alpha=0), \\
l+m+n+j+2=0 & (\alpha=\omega / 2),\end{cases} \\
\sum_{l, m}^{(4)} & = \begin{cases}l+m+1=0 & (\alpha=0), \\
l+m+2=0 & (\alpha=\omega / 2),\end{cases}
\end{aligned}
$$




$$
\begin{aligned}
& \sum_{l, m}^{(5)}=\left\{\begin{array}{l}
l+m-1=0 \quad(\alpha=0), \\
l+m=0 \quad(\alpha=\omega / 2),
\end{array}\right. \\
& \sum_{l, m}^{(6)}= \begin{cases}l+m+M_{1}=0 & (\alpha=0), \\
l+m+M_{1}+1=0 & (\alpha=\omega / 2),\end{cases} \\
& \sum_{l, m}^{(7)}= \begin{cases}l+m-M_{1}=0 & (\alpha=0), \\
l+m-M_{1}+1=0 & (\alpha=\omega / 2),\end{cases} \\
& \sum_{l, m}^{(8)}= \begin{cases}l+m+M_{2}=0 & (\alpha=0), \\
l+m+M_{2}+1=0 & (\alpha=\omega / 2),\end{cases} \\
& \sum_{l, m}^{(9)}= \begin{cases}l+m-M_{2}=0 & (\alpha=0), \\
l+m-M_{2}+1=0 & (\alpha=\omega / 2) .\end{cases}
\end{aligned}
$$

Note that terms involving the derivative of the pressure with respect to $z$ appear in the solvability condition. These may be computed from the flow variables, as discussed in Appendix D.

\section{Appendix C. Cubic coefficient for hexagons.}

$$
\begin{aligned}
\gamma_{3}= & \sum_{l, m, n}^{(2)} Z_{l}^{*}\left(2 D W_{1, m}(0) Z_{2,2, n}-2 Z_{1, m} D W_{2,2, n}(0)\right) \\
& +\sum_{l, m, n}^{(2)} W_{l}^{*}(0)\left(2 D^{2} W_{1, m}(0) W_{2,2, n}(0)-2 W_{1, m}(0) D^{2} W_{2,2, n}(0)\right) \\
& +4 k^{2} \sum_{l, m, n}^{(2)} W_{l}^{*}(0)\left(D P_{1, m}(0) Z_{2,2, n}(0)+Z_{1, m} D P_{2,2, n}(0)+Z_{1, m} D P_{2,0, n}(0)\right. \\
& \left.+\frac{1}{3} Z_{1, m} D P_{2,3, n}(0)\right) \\
& -C k^{2} \sum_{l, m, n}^{(2)} W_{l}^{*}(0)\left(4 Z_{1, m} D^{2} W_{2,2, n}(0)+8 D^{2} W_{1, m}(0) Z_{2,2, n}\right) \\
& -\sum_{l, m, n}^{(2)} W_{l}^{*}(0)\left(-12 C k^{4} Z_{1, m} W_{2,2, n}(0)+2 D W_{1, m}(0) D W_{2,2, n}(0)\right) \\
& +C \sum_{l, m, n}^{(2)} D W_{l}^{*}(0)\left(2 D^{3} W_{1, m}(0) Z_{2,2, n}-2 Z_{1, m} D^{3} W_{2,2, n}(0)\right) \\
& +C \sum_{l, m, n}^{(2)} D W_{l}^{*}(0)\left(4 k^{2} Z_{1, m} D W_{2,2, n}(0)-16 k^{2} D W_{1, m}(0) Z_{2,2, n}\right) \\
& -4 \sum_{l, m, n, j}^{(3)} Z_{l}^{*} Z_{1, m} Z_{1, n} D^{2} W_{1, j}(0) \\
& +k^{2} \sum_{l, m, n, j}^{(3)} W_{l}^{*}(0) Z_{1, m} Z_{1, n}\left(12 D^{2} P_{1, j}(0)+36 C k^{2} D W_{1, j}(0)-8 C D^{3} W_{1, j}(0)\right. \\
& \left.+6 Z_{1, j}\right)
\end{aligned}
$$




$$
\begin{aligned}
& -C \sum_{l, m, n, j}^{(3)} D W_{l}^{*}(0) Z_{1, m} Z_{1, n}\left(24 k^{2} D^{2} W_{1, j}(0)+4 D^{4} W_{1, j}(0)\right) \\
& -\sum_{l, m, n}^{(2)} \int_{-h / l}^{0} W_{l}^{*}(z)\left(10 k^{2} D W_{1, m}(z) W_{2,2, n}(z)+6 k^{2} W_{1, m}(z) D W_{2,2, n}(z)\right) \\
& +4 \sum_{l, m, n}^{(2)} \int_{-h / l}^{0} W_{l}^{*}(z) D W_{1, m}(z) D^{2} W_{2,2, n}(z) \\
(\mathrm{C} .1) \quad+ & \sum_{l, m, n}^{(2)} \int_{-h / l}^{0} W_{l}^{*}(z)\left(2 W_{1, m}(z) D^{3} W_{2,2, n}(z)-2 D^{3} W_{1, m}(0) W_{2,2, n}(z)\right)
\end{aligned}
$$

Appendix D. A posteriori computation of the pressure. We compute the pressure from the Navier-Stokes equations. As we need only the derivative of the pressure with respect to $z$, we need only consider the third component of the Navier-Stokes equations:

$$
\partial_{z} p=-\partial_{t} w+C \Delta w-\mathbf{u} \cdot \nabla w .
$$

At the first order this reduces to

$$
\partial_{z} p_{1}=-\partial_{\tau} w_{1}+C \Delta w_{1}
$$

and then we can take $\partial_{z} p_{1}$ in the form

$$
\partial_{z} p_{1}=A_{S}\left(e^{i k x}+e^{-i k x}\right) \sum_{n} D P_{1, n}(z) e^{i(n \omega+\alpha) \tau},
$$

or the equivalent form for rectangles and hexagons, where $D P_{1, n}(z)$ solves

$$
D P_{1, n}(z)=\left[-i(n \omega+\alpha)+C\left(D^{2}-k^{2}\right)\right] W_{1, n}(z) .
$$

At the second order we have

$$
\partial_{z} p_{2}=-\partial_{\tau} w_{2}+C \Delta w_{2}-\mathbf{u}_{1} \cdot \nabla w_{1}-\partial_{T_{1}} w_{1} .
$$

The solution for the rectangular pattern does not depend on $T_{1}$. Here we take $\partial_{z} p_{2}$ in the form

$$
\begin{aligned}
\partial_{z} p_{2}= & A_{R}^{2}\left[e^{2 i k x}+e^{2 i k(c x+s y)}+\text { c.c. }\right] \sum_{n} D P_{2,1, n}(z) e^{i(n \omega+2 \alpha) \tau} \\
& +A_{R}^{2}\left[e^{i k[(1+c) x+s y]}+\text { c.c. }\right] \sum_{n} D P_{2,2, n}(z) e^{i(n \omega+2 \alpha) \tau} \\
& +A_{R}^{2}\left[e^{i k[(1-c) x-s y]}+\text { c.c. }\right] \sum_{n} D P_{2,3, n}(z) e^{i(n \omega+2 \alpha) \tau} \\
& +A_{R}^{2} \sum_{n} D P_{2,4, n}(z) e^{i(n \omega+2 \alpha) \tau}
\end{aligned}
$$

where

$$
D P_{2,1, n}(z)=\left[-i(n \omega+2 \alpha)+C\left(D^{2}-4 k^{2}\right)\right] W_{2,1, n}(z),
$$




$$
\begin{aligned}
D P_{2,2, n}(z)= & {\left[-i(n \omega+2 \alpha)+C\left(D^{2}-2(1+c) k^{2}\right)\right] W_{2,2, n}(z) } \\
& -2(1-c) \sum_{(l+m=n)} W_{1, l}(z) D W_{1, m}(z), \\
D P_{2,3, n}(z)= & {\left[-i(n \omega+2 \alpha)+C\left(D^{2}-2(1-c) k^{2}\right)\right] W_{2,3, n}(z) } \\
& -2(1+c) \sum_{(l+m=n)} W_{1, l}(z) D W_{1, m}(z), \\
D P_{2,4, n}(z)= & -8 \sum_{(l+m=n)} W_{1, l}(z) D W_{1, m}(z) .
\end{aligned}
$$

In the hexagonal pattern, the solution depends on both $T_{1}$ and $T_{2}$. We take the pressure as the sum of two terms:

$$
\partial_{z} p_{2}=\partial_{z} \hat{p}_{2}+\partial_{z} \tilde{p}_{2}
$$

where $\hat{p}_{z}$ solves

$$
\partial_{z} \hat{p}_{2}=-\partial_{\tau} w_{2}+C \Delta w_{2}-\mathbf{u}_{1} \cdot \nabla w_{1},
$$

while $\tilde{p}_{z}=-\partial_{T_{1}} w_{1}$. We take $\hat{p}_{z}$ in the form of a hexagonal pattern:

$$
\begin{aligned}
\partial_{z} \hat{p}_{2}= & A_{H}^{2}\left[e^{i k x}+e^{i k(-x+\sqrt{3} y) / 2}+e^{i k(-x-\sqrt{3} y) / 2}+c . c .\right] \sum_{n} D P_{2,0, n}(z) e^{i(n \omega+2 \alpha) \tau} \\
& +A_{H}^{2}\left[e^{2 i k x}+e^{i k(-x+\sqrt{3} y)}+e^{i k(-x-\sqrt{3} y)}+c . c .\right] \sum_{n} D P_{2,1, n}(z) e^{i(n \omega+2 \alpha) \tau} \\
& +A_{H}^{2}\left[e^{i k \sqrt{3} y}+e^{i k(3 x+\sqrt{3} y) / 2}+e^{i k(3 x-\sqrt{3} y) / 2}+c . c .\right] \sum_{n} D P_{2,2, n}(z) e^{i(n \omega+2 \alpha) \tau} \\
& +A_{H}^{2} \sum_{n} D P_{2,3, n}(z) e^{i(n \omega+2 \alpha) \tau},
\end{aligned}
$$

where

$$
\begin{aligned}
D P_{2,0, n}(z)= & -3 \sum_{(l+m=n)} W_{1, l}(z) D W_{1, m}(z) \\
D P_{2,1, n}(z)= & {\left[-i(n \omega+2 \alpha)+C\left(D^{2}-4 k^{2}\right)\right] W_{2,1, n}(z) } \\
D P_{2,2, n}(z)= & {\left[-i(n \omega+2 \alpha)+C\left(D^{2}-3 k^{2}\right)\right] W_{2,2, n}(z) } \\
& -\sum_{(l+m=n)} W_{1, l}(z) D W_{1, m}(z) \\
D P_{2,3, n}(z)= & -12 \sum_{(l+m=n)} W_{1, l}(z) D W_{1, m}(z)
\end{aligned}
$$

Acknowledgments. We have benefited from discussions with a number of people during the course of writing this paper, including Paul Matthews, Alastair Rucklidge, and José Vega. We are very grateful to Peilong Chen for letting us use his code to compare our work with his and to the anonymous referees, who made a number of helpful suggestions.

\section{REFERENCES}

[1] M. FARADAY, On the forms and states assumed by fluids in contact with vibrating elastic surfaces, Phil. Trans. Roy. Soc. Lond., 121 (1831), pp. 319-340. 
[2] S. Ciliberto and J. P. Gollub, Chaotic mode competition in parametrically forced surface waves, J. Fluid Mech., 158 (1985), pp. 381-398.

[3] W. S. Edwards and S. FAuve, Patterns and quasi-patterns in the Faraday experiment, J. Fluid Mech., 278 (1994), pp. 123-148.

[4] A. Kudrolli, B. Pier, And J. P. Gollub, Superlattice patterns in surface waves, Phys. D, 123 (1998), pp. 99-111.

[5] H. Arbell and J. Fineberg, Spatial and temporal dynamics of two interacting modes in parametrically driven surface waves, Phys. Rev. Lett., 81 (1998), pp. 4384-4387.

[6] H. Arbell and J. Fineberg, Two-mode rhomboidal states in driven surface waves, Phys. Rev. Lett., 84 (2000), pp. 654-657.

[7] H. Arbell and J. Fineberg, Temporally harmonic oscillons in Newtonian fluids, Phys. Rev. Lett., 85 (2000), pp. 756-759.

[8] D. Binks AND W. VAN DeR Water, Nonlinear pattern formation of Faraday waves, Phys. Rev. Lett., 78 (1997), pp. 4043-4046.

[9] P. H. Wright and J. R. Saylor, Patterning of particulate films using Faraday waves, Rev. Sci. Instrum., 74 (2003), pp. 4063-4070.

[10] J. R. Saylor And A. L. Kinard, Simulation of particle deposition beneath Faraday waves in thin liquid films, Phys. Fluids, 17 (2005), 047107.

[11] J. R. SAYlOR AND R. A. HANDler, Gas transport across an air/water interface populated with capillary waves, Phys. Fluids, 9 (1997), pp. 2529-2541.

[12] T. G. Leighton, From sea to surgeries, from babbling brooks to baby scans: Bubble acoustics at ISVR, Proc. Inst. Acoustics, 26 (2004), pp. 357-381.

[13] T. B. Benjamin and F. Unsell, The stability of a plane free surface of a liquid in vertical periodic motion, Proc. Roy. Soc. London Ser. A, 225 (1954), pp. 505-515.

[14] K. Kumar and L. Tuckerman, Parametric instability of the interface between two fluids, J. Fluid Mech., 279 (1994), pp. 49-68.

[15] T. Besson, W. S. Edwards, and L. S. Tuckerman, Two-frequency parametric excitation of surface waves, Phys. Rev. E (3), 54 (1996), pp. 507-513.

[16] W. Zhang and J. Viñals, Pattern formation in weakly damped parametric surface waves, J. Fluid Mech., 336 (1997), pp. 301-330.

[17] W. Zhang AND J. Viñals, Square patterns and quasipatterns in weakly damped Faraday waves, Phys. Rev. E (3), 53 (1996), pp. R4283-R4286.

[18] M. Silber, C. Topaz, And A. C. Skeldon, Two-frequency forced Faraday waves: Weakly damped modes and pattern selection, Phys. D, 143 (2000), pp. 205-225.

[19] C. M. Topaz AND M. Silber, Resonances and superlattice pattern stabilization in twofrequency forced Faraday waves, Phys. D, 172 (2002), pp. 1-29.

[20] J. Porter AND M. Silber, Broken symmetries and pattern formation in two-frequency forced Faraday waves, Phys. Rev. Lett., 89 (2002), 084051.

[21] C. Martel and E. Knobloch, Damping of nearly inviscid water waves, Phys. Rev. E (3), 56 (1997), pp. 5544-5548.

[22] P. Chen AND J. Viñals, Amplitude equation and pattern selection in Faraday waves, Phys. Rev. E (3), 60 (1999), pp. 559-570.

[23] F. J. Mancebo and J. M. Vega, Viscous Faraday waves in two-dimensional large-aspect-ratio containers, J. Fluid Mech., 560 (2006), pp. 369-393.

[24] A. M. Rucklidge, Pattern formation in large domains, R. Soc. Lond. Philos. Trans. Ser. A Math. Phys. Eng. Sci., 361 (2003), pp. 2649-2664.

[25] B. Dionne and M. Golubitsky, Planforms in two and three dimensions, Z. Angew. Math. Phys., 43 (1992), pp. 36-62.

[26] B. Dionne, M. Silber, And A. C. Skeldon, Stability results for steady, spatially periodic planforms, Nonlinearity, 10 (1997), pp. 321-353.

[27] M. Silber AND M. R. E. Proctor, Nonlinear competition between small and large hexagonal patterns, Phys. Rev. Lett., 81 (1998), pp. 2450-2453.

[28] A. C. Skeldon And M. Silber, New stability results for patterns in a model of long-wavelength convection, Phys. D, 122 (1998), pp. 117-133.

[29] S. L. Judd And M. Silber, Simple and superlattice Turing patterns in reaction-diffusion systems: Bifurcation, bistability and parameter collapse, Phys. D, 136 (2000), pp. 45-65.

[30] M. Golubitsky, J. W. Swift, and E. Knobloch, Symmetries and pattern selection in Rayleigh-Bénard convection, Phys. D, 10 (1984), pp. 249-276.

[31] D. Tse, A. M. Rucklidge, R. Hoyle, and M. Silber, Spatial period-multiplying instabilities of hexagonal Faraday waves, Phys. D, 146 (2000), pp. 367-387.

[32] T. Epstein And J. FineBerg, Grid states and nonlinear selection in parametrically excited surface waves, Phys. Rev. E (3), 73 (2006), 055302. 